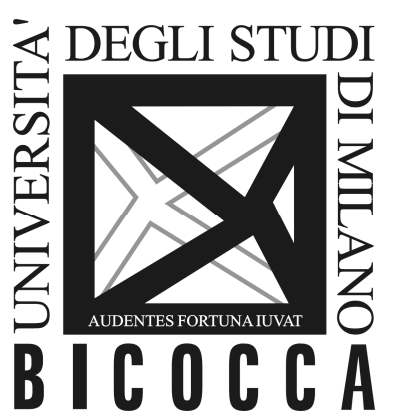

\author{
DEPARTMENT OF ECONOMICS, \\ MANAGEMENT AND STATISTICS \\ UNIVERSITY OF MILAN - BICOCCA
}

DEMS WORKING PAPER SERIES

Existence, multiplicity and dynamic complexity in an OLG model with fiscal policy and debt

Lorenzo Cerboni Baiardi, Ahmad Naimzada

No. 385 - June 2018

Dipartimento di Economia, Metodi Quantitativi e Strategie di Impresa Università degli Studi di Milano - Bicocca

http://dems.unimib.it/ 


\title{
EXISTENCE, MULTIPLICITY AND DYNAMIC COMPLEXITY IN AN OLG MODEL WITH FISCAL POLICY AND DEBT.
}

\author{
Lorenzo Cerboni Baiardi, Ahmad Naimzada \\ Department of Economics, Quantitative Methods and Management, University of Milano - Bicocca, \\ 20126 - Milano, Italy
}

ABstract. We consider the overlapping generation model formulated in Dioikitopoulos (2018) that tackles the problem of fiscal policy rules for debt sustainability, allowing for the presence of debt bubbles. The author gives conditions for sustainability achievement in terms of debt and capital control channels, taking into account initial conditions. Our mathematical analysis improves his study and reveals a wider spectrum of possible economic outcomes that might sometimes be opposed to the conclusions to which Dioikitopoulos (2018) comes. In detail, we reconsider the role of fiscal policy prescriptions, implemented by means of debt and capital responsiveness parameters, in determining the existence and multiplicity of stationary states. We also deepen the influence of policy parameters on local stability properties, highlighting the possible occurrence of two bifurcation scenarios and the consequent emergence of periodic and complex dynamics. Moreover, we review the role of fiscal policies in changing the fate of incoming economic scenarios and in preventing non sustainable paths from occurring.

Keywords: fiscal sustainability, fiscal rules, bond financed deficit, local stability, bifurcations, basins of attraction, dynamic complexities.

JEL classification: C6, E6, H6, H30 


\section{INTRODUCTION}

In the recent macroeconomic literature numerous efforts have been proposed with the aim to provide theoretical frameworks of fiscal policy rules for debt sustainability allowing for the presence of debt bubbles. The significant attention on the sustainability of fiscal policies should be ascribed to continuous adaptations, especially in Europe, of debt control policies and, also, to the unexpected circumstance according to which similar fiscal measures implemented in similar countries have produced different economic outcomes (see to this purpose [5]). Within this research stream, the interesting contribution by [7] is placed, where the author formulates a suitable model to tackle the problem concerning debt sustainability and debt bubbles.

Through the model proposed in [7], the author suggests that countries may display different responses under similar fiscal policy measures due to different initial economic configurations, described by means of inherited debt and capital levels, resulting from their historical experiences. Indeed, incoming economic scenarios may result in sustainable paths depending on initial conditions: if initial capital and debt levels are included within the set of points of the phase space iterated in the long run towards a stable attractor, sustainability is achieved. Differently, if initial conditions belong to the set of points giving rise to divergent trajectories, the economy follows non sustainable paths. In particular, through numerical simulations, the author suggests that, given a certain level of inherited debt, sustainability is always achieved given sufficiently high levels of initial capital. We show that this finding is true when limited to a local view and conditioned upon the stability properties of equilibria. In addition, we remark that extending such a local behavior to a global perspective is not recommendable, being the model characterized by strong nonlinearities.

In [7, the author's remarks on normative aspects are based on the fiscal rule he considers, according to which two responsiveness parameters balance the relevance of debt and capital levels, such that deficit is determined accordingly. The author suggests that the fiscal policy authority interested in sustainability should act on debt and capital control channels, by increasing debt sensitivity or decreasing capital sensitivity, thus expecting a stable stationary state to rise and initial conditions to be placed within the basin of attraction of sustainable paths. 
However, we note that this conclusion is affected by substantial flaws that limit its correctness only to particular circumstances. Indeed, at first, the author obtains unsuitable conditions for the existence of stationary states in terms of fiscal policy parameters. Moreover, the author evaluates the local stability of stationary states using the so called "arrows method", which, depending on the sign of state variables variations across regions of the phase space, gives insights on dynamic behaviors of trajectories. However, in our opinion, this methodological approach led the author to obtain misleading results, being it applied to a discrete time dynamical system. Indeed, as noted in [2], "qualitative information drown from discrete phase diagrams is quite tentative and should be confirmed from the local information contained in Jacobian matrices". Finally, the author does not considers the joint effects that the mentioned fiscal policy has on both the stability properties of stationary states and the shape and the extension of basins of attraction of sustainable trajectories.

This motivates us to improve the analysis performed by [7]. Our contribution aims to deepen the implications of the model by means of a rigorous and structured mathematical analysis, performed through analytical and numerical methods. The analysis reveals a wider spectrum of possible economic outcomes that might sometimes be opposed to the conclusions to which [7] comes.

In detail, we distinguish within our analysis, those results related with the descriptive part of the model and those related with normative aspects, namely fiscal policy prescriptions, considered in [7]. As for the first part, we derive the analytic expressions of the two stationary states of the model and we prove, in a particular case, that their appearance is associated with the occurrence of saddle-node bifurcation. However, the coincidence of saddle-node bifurcation with the appearance of stationary state is a robust scenario found in wide parameters configurations. Moreover, we also give necessary and sufficient conditions for the componentwise positiveness of stationary states which, in general, may describe economic equilibria characterized by negative debt levels. The local stability analysis, performed by means of analytical as well as numerical tools, reveals that the stationary states may emerge as a saddle and a stable node. Hence, when this is the case, the economy follows a sustainable path as the convergence towards the stable equilibrium occurs. In addition, 
the analysis reveals that the two equilibria may also be simultaneously unstable. This is possible since, just after their appearance through saddle-node bifurcation, they may emerge as an unstable node and a saddle. The same scenario may also result from the loss of stability of the stable stationary state through period-doubling bifurcation. In both cases, it is still possible for dynamic sustainability patterns to occur as economic dynamics take place along bounded trajectories, consisting of periodic or chaotic orbits. Otherwise, even in the presence of stationary states, it may happen that all the attractors are unstable and non sustainability is the unique outcome of the model.

In addition, we deepen the aspects concerning the dependence of incoming scenarios on initial economic configurations, described in terms of inherited debt and capital levels. Differently from what has been found in [7, we show, through exemplary numerical simulations, the wide variety of possible consequences that different initial conditions may produce.

As for the implications of fiscal policy prescriptions suggested in [7], we show that, as they are implemented, neither the presence of a stable stationary state nor a stable attracting set is ensured. In detail, we provide necessary and sufficient conditions, expressed in terms of fiscal parameters, for the existence of stationary states as well as for their componentwise positiveness. Moreover, we show that fiscal parameters influence the stability properties of the stationary states. They are capable to cause, besides the above mentioned saddle-node bifurcations, period-doubling bifurcations and they may give rise to periodic and chaotic trajectories after the usual period-doubling cascade. In addition, further reinforcements of such policies, may determine the loss of stability of all the attractors and, as a consequence, non sustainable scenarios occur anyway, regardless of whichever the initial economic configurations is. Finally, along the line marked by [7], we recognize the importance of the fiscal policy in determining the extension and shape of basins of attraction of sustainability. This is a remarkable effect that changes the fate of economic dynamics starting from initial conditions that would have led to non sustainability if no action had been taken by the authority. However, fiscal policy implemented by increasing (decreasing) debt (capital) sensitivity is not so effective to allow the authority to prevent non sustainability paths from occurring. Indeed, as responsiveness parameters are adapted accordingly, both the attractor changes its stability properties and its basin undergoes changes 
of different nature. This joint effects may result in including some initial conditions within the basin of sustainability and, simultaneously, in excluding other initial conditions from it. Also, the basin of sustainability should undergo to structural changes: it may reduce in favor of the widening of the diverging trajectories' basin and, as final outcome, it may disappear as soon as the attractor loses its stability.

The paper is organized as follows. In Section 2 we retrieve the model formulation proposed in [7]. In Section 3 we give explicit expressions of stationary states of the model and we provide necessary and sufficient conditions for their existence as well as for their componentwise positiveness. In Section 4 we consider the occurrence of two bifurcation scenarios determined by policy parameters. In Section 5 we deepen the structure of basins of sustainable paths and their variations due to fiscal policy actions. Section 6 concludes.

\section{THE MODEL}

We recall the overlapping generation model proposed in [7, together its formulation.

2.1. Demand side. Following [6] and [1], the author considers $N_{t}$ two-period living consumers, who choose their consumptions $C_{t}$ and $d_{t+1}$ in their first and second life period respectively, in order to maximize the intertemporal utility

$$
U=\ln C_{t}+\beta \ln d_{t+1}
$$

where $\beta>0$ represents the importance that agents gives to their section life period utility. In the first period, agents are active workers that inelastically supply labor receiving the wage $w_{t}$ taxed at the amount $\tau_{t}<1$. In the second life period, agents consume their savings and receive a return $r_{t+1}$ on their savings $S$. Savings, in turn, are determined by solving the intertemporal maximization problem and are given in terms of the after-tax wage rate and the saving propensity $s=\beta /(\beta+1)$ :

$$
S\left(w_{t}\right)=s\left(1-\tau_{t}\right) w_{t}
$$

2.2. Supply side. It is considered a continuum of firms that produce according to the Cobb-Douglas function, using capital $k_{t}$, labor $l_{t}$ and a public expenditure $g_{t}$. Hence, the industry output $y_{t}$ results

$$
y_{t}=A k^{\alpha} l_{t}^{1-\alpha} g_{t}^{\gamma}, \alpha+\gamma<1
$$


where $\alpha>0$ denotes the share of physical capital on the production function and $\gamma>0$ denotes the share $\gamma$ of public spending on production. The latter requirement, $\gamma>0$, follows from empirical facts as presented in [1], [8], and [4. The wage rate $w_{t}$ and the real return on capital $R_{t}$, using the labor market clearing condition $l_{t}=1$, are determined by

$$
w_{t}=(1-\alpha) A k_{t}^{\alpha} g_{t}^{\gamma}
$$

and

$$
R_{t}=\alpha A k^{\alpha-1} g_{t}^{\gamma}
$$

2.3. Government. Following [9] and [12], the author assumes that the supply of the public good is determined by a production efficiency rule in the spirit of [10], according to which the marginal benefit generated by the public expenditures must be equal to the marginal cost of its production:

$$
g_{t}=\left(\gamma A k_{t}^{\alpha}\right)^{\frac{1}{1-\gamma}}
$$

According to (1), government expenditures adjust through time subject to the level of the capital stock, so that the marginal benefit of public funds decreases as capital increases. Using (1), the author computes the equilibrium wage and real interest rate, respectively, as

$$
w\left(k_{t}\right)=(1-\alpha) A k^{\alpha} g_{t}^{\gamma}
$$

and

$$
R\left(k_{t}\right)=\alpha A k^{\alpha-1} g_{t}^{\gamma}
$$

Moreover, the author assumes that the government finances public expenditures from taxation as well as by issuing government debt $B_{t}$. Hence, the government's budget constraint results

$$
B_{t+1}=R_{t} B_{t}+g_{t}-\tau_{t} w_{t}
$$

Following the fiscal rule estimated by [3], the author considers that the primary surplus/deficit can be expressed as a function of the level of debt and income and determined by the fiscal policy parameters responsiveness parameters $a>$ 0 and $b>0$ as

$$
g_{t}-\tau_{t} w_{t}=-a B_{t}+b y_{t}
$$

Policy parameter $a$ and $b$ measure the sensitivity of the deficits to the level of debt and income respectively. 
2.4. Dynamic equations. As shown in [7], at equilibrium, where savings equal investments in real capital and government bonds, the dynamic equilibrium is determined by the following recurrences

$$
T:\left\{\begin{array}{l}
k_{t+1}=(s(1-\alpha)+s(b-\gamma)-b) y\left(k_{t}\right)+\left(a(1-s)-R\left(k_{t}\right)\right) B_{t} \\
B_{t+1}=\left(R\left(k_{t}\right)-a\right) B_{t}+b y\left(k_{t}\right)
\end{array}\right.
$$

\section{Existence, multiplicity And COMPONENTWise POSITIVENESS OF}

\section{STATIONARY STATES}

In this Section we improve the results obtained in [7] by providing analytical expressions of stationary states of the map (2) in terms of parameters as well as necessary and sufficient conditions for their existence. Moreover, we note that, in [7], the author does not consider the possibility of negative debt

equilibrium levels. Hence, we perfect his analysis by further deriving necessary and sufficient conditions for componentwise positiveness of stationary states.

For brevity's sake, let us introduce the following shortcuts:

$$
\begin{aligned}
& \delta_{1}=\alpha+s(1-\alpha-\gamma)(1+a)-b(1-s) \\
& \delta_{2}=4 \alpha(1+a)(s(1-\alpha-\gamma)+b s) \\
& \Delta=\delta_{1}^{2}-\delta_{2}
\end{aligned}
$$

Proposition 3.1. The following cases occur.

i) Assume $a(1-s)(s(1-\alpha)+s(b-\gamma)-b) \neq \alpha$. Then, the map $T$ has two stationary states $E_{-}=\left(k_{-}, B_{-}\right)$and $E_{+}=\left(k_{+}, B_{+}\right)$, with $k_{-}, k_{+}>0$, if and only if

$$
\sqrt{\delta_{2}} \leq \delta_{1}
$$

Analytical expressions of $E_{+}$and $E_{-}$are

$$
\begin{aligned}
& E_{+}=\left(k_{+}, B_{+}\right)=\left(1, \frac{b r_{+}}{\alpha\left(1+a-r_{+}\right)}\right) \cdot\left(\frac{r_{+}}{\alpha A(\gamma A)^{\frac{\gamma}{1-\gamma}}}\right)^{-\frac{1-\gamma}{1-\alpha-\gamma}} \\
& E_{-}=\left(k_{-}, B_{-}\right)=\left(1, \frac{b r_{-}}{\alpha\left(1+a-r_{-}\right)}\right) \cdot\left(\frac{r_{-}}{\alpha A(\gamma A)^{\frac{\gamma}{1-\gamma}}}\right)^{-\frac{1-\gamma}{1-\alpha-\gamma}}
\end{aligned}
$$

where

$$
r_{ \pm}=\frac{1}{2(1+a)(\alpha+s(1-\alpha-\gamma)+b s)}\left(\delta_{1} \pm \sqrt{\delta_{1}^{2}-\delta_{2}}\right)
$$


In addition, provided that (3) holds, $E_{-}$and $E_{+}$are componentwise positive if and only if

$$
\delta_{1}<\frac{\delta_{2}}{2 \alpha}
$$

ii) Assume $a(1-s)(s(1-\alpha)+s(b-\gamma)-b) \neq \alpha$. Then $E_{-}$is the unique componentwise positive stationary state of $T$.

Proof. See Appendix 7.1.

The following Corollary highlights the role of policy parameters $a$ and $b$ in determining existence and componentwise positiveness of stationary states and it follows from rewriting relations (3) and (7) in terms of $a$ and $b$.

Corollary 3.2. Assume $a(1-s)(s(a-\alpha)+s(b-\gamma)-b) \neq \alpha$. Then the following cases occur.

i) Stationary states $E_{+}=\left(k_{+}, B_{+}\right)$and $E_{-}=\left(k_{-}, B_{-}\right)$, with $k_{+}, k_{-}>0$, exist if and only if

$$
a \leq a_{-} \text {or } a_{+} \leq a
$$

where

$$
\begin{aligned}
a_{ \pm}= & \frac{1}{s(1-\alpha-\gamma)^{2}}(\alpha(1+2 b-\alpha-\gamma)+b(1-s)(1-\alpha-\gamma) \pm \\
& \left.\quad \pm \sqrt{(\alpha(1+2 b-\alpha-\gamma)+b(1-s)(1-\alpha-\gamma))^{2}-(1-\alpha-\gamma)^{2}(\alpha-b(1-s))^{2}}\right)-1
\end{aligned}
$$

Moreover, $E_{-}$and $E_{+}$are componentwise positive if and only if

$$
a_{+} \leq a
$$

ii) Stationary states $E_{+}=\left(k_{+}, B_{+}\right)$and $E_{-}=\left(k_{-}, B_{-}\right)$, with $k_{+}, k_{-}>0$, exist if and only if

$$
b \leq b_{\max }
$$

where

$$
\begin{aligned}
b_{\max }:=\frac{\alpha}{1-s} & +\frac{s(1-\alpha-\gamma)(1+a)}{1-s}+\frac{2 \alpha s(1+a)}{(1-s)^{2}}+ \\
& -\frac{2}{(1-s)^{2}} \sqrt{\alpha s(1+a)(1+a s)(\alpha+(1-s)(1-\alpha-\gamma))}
\end{aligned}
$$

Moreover, $E_{-}$and $E_{+}$are componentwise positive if and only if

$$
b_{\min } \leq b \leq b_{\max }
$$


where

$$
b_{\min }:=\frac{\alpha-s(1-\alpha-\gamma)(1+a)}{1-s+2 s(1+a)}
$$

Proof. See Appendix 7.2.

Remark 3.3. In the remainder of the paper, we will denote $a_{+}$as $a_{\min }$.

Relation 8 provides necessary and sufficient conditions for the existence of stationary states expressed in terms of debt responsiveness $a$ and refines the result obtained by [7] in Proposition 1, where values of $a$, which are claimed to assure the existence of $E_{+}$and $E_{-}$, should not exceed an upper threshold. In addition, relation (8) is combined with relation $(10)$ and together provide necessary and sufficient conditions for componentwise positiveness of stationary states requiring values of $a$ not to lie below the threshold $a_{\text {min }}$. Examples of different configurations, occurring at increasing values of $a$, are provided in figures 1 and 2 . In those figures, locus $\phi$ and $\psi$, which correspond respectively to the curves of null variations of the state variables $k$ and $B$, are shown together with their intersections matching with the stationary states $E_{+}$and $E_{-}$. Explicit expressions of $\phi$ and $\psi$ are provided in 15. In particular, figure 1 shows that increasing values of $a$ lead to the appearance of $E_{+}$and $E_{-}$, thus providing a counterexample to Proposition 1 in [7]. In addition, figure 2 shows the possibility for stationary states to lie in the region of negative debt levels.
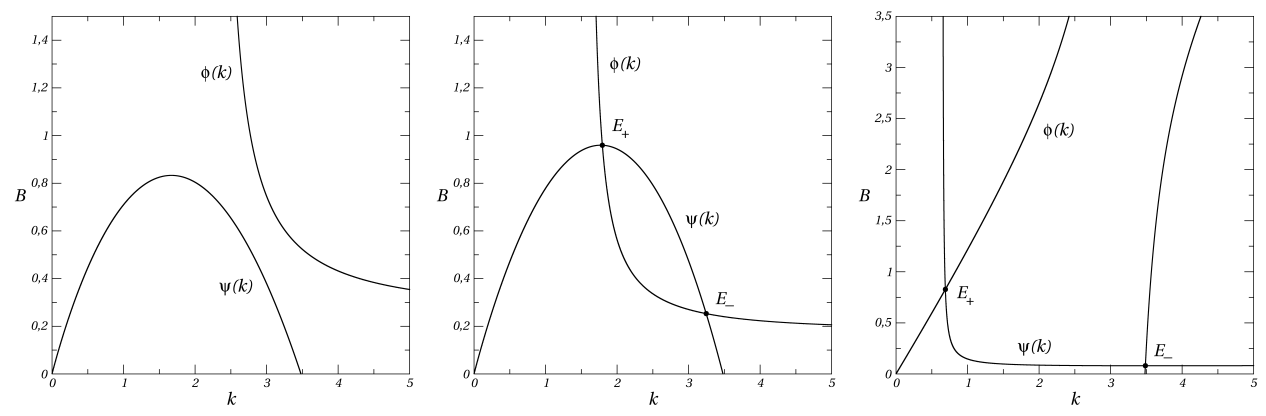

Figure 1. Locus $\phi(k)$ and $\psi(k)$ and their intersection $E_{-}$and $E_{+}$, when existing, at $a=0.15$ (left), $a=0.5$ (center) and $a=1.8$ (right). Other parameters are $b=0.013, \alpha=0.25$, $\gamma=0.15, \beta=0.99$ and $A=8$. Also, $a_{-}=-0.474442$ and $a_{\text {min }}=0.265884$. 

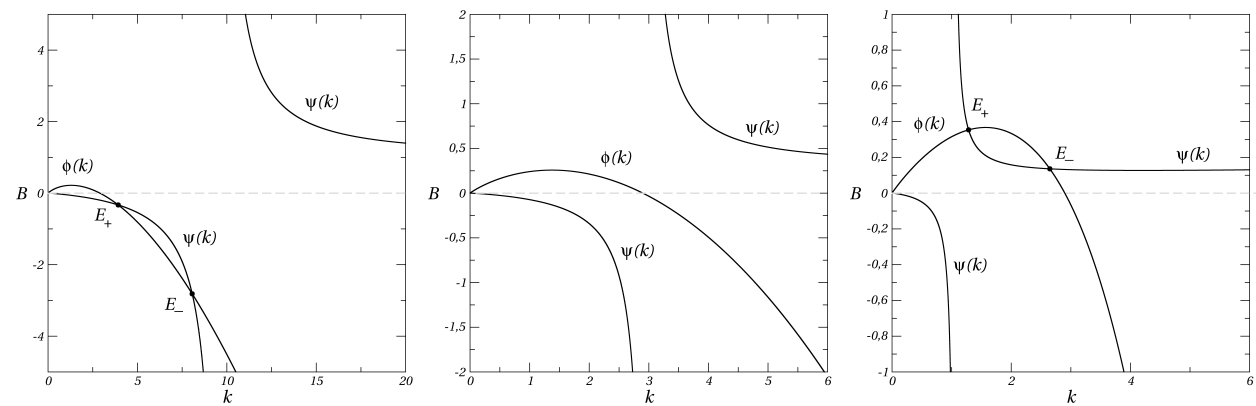

Figure 2. Locus $\phi(k)$ and $\psi(k)$ and their intersection $E_{-}$and $E_{+}$, when existing, at $a=0.1$ (left), $a=1$ (center) and $a=2.4$ (right). Other parameters are $b=0.013, \alpha=0.42, \gamma=0.15$, $\beta=0.99$ and $A=8$. Also, $a_{-}=0.259772$ and $a_{\min }=1.96544$.

Moreover, relation (11) provides necessary and sufficient conditions for the existence of stationary states expressed in terms of capital responsiveness $b$, by requiring values of $b$ to lie below a certain threshold $b_{\max }$ without crossing the lower bound $b_{\min }$. This result perfects the one given in [7, also with a different analytical expression for $b_{\max }$. In addition, relation (11) is combined with relation (13) and together provide necessary and sufficient conditions for the existence and componentwise positiveness of $E_{+}$and $E_{-}$. An example of different configurations, occurring at increasing values of $b$, are provided in figure 2.
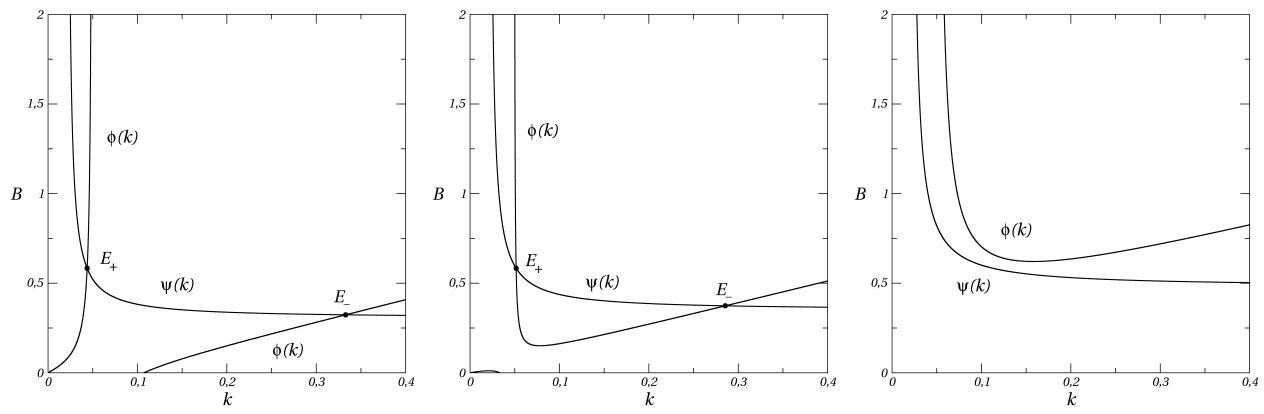

Figure 3. Locus $\phi(k)$ and $\psi(k)$ and their intersection points $E_{-}$and $E_{+}$, when existing, at $b=0.07$ (left), $b=0.08$ (center) and $b=0.11$ (right). Other parameters are $a=0.9, \alpha=0.005$, $\gamma=0.15, \beta=0.1$ and $A=8$. Also, $b_{\min }=-0.112355$ and $b_{\max }=0.103097$. 
Remark 3.4. Condition (10) results from rewriting relations (3) and (7) in terms of the fiscal rule responsiveness parameter a. Similarly, condition (13) results from rewriting the same relations (3) and (7) in terms of the fiscal rule responsiveness parameter $b$. Hence, (10) and (13) are equivalent conditions being, each on its own, necessary and sufficient for the existence and componentwise positiveness of the stationary states $E_{-}$and $E_{+}$. It follows that, at any fixed $b>0$, the relation $a \geq a_{\min }$ implies $b_{\max } \geq b$ and $b_{\min } \leq b$. Conversely, at any fixed $a>0$, the relation $b_{\min } \leq b \leq b_{\max }$ implies $a_{\min } \leq a$.

As follows from remark 3.4, conditions on a single fiscal parameter between $a$ and $b$ can be imposed to ensure the existence of stationary states $E_{-}$and $E_{+}$which, in the present model, is a prerequisite for economic dynamics to converge towards an attractor. Indeed, besides the existence of $E_{+}$and $E_{-}$, also initial conditions as well as local stability features of attractors determine feasible outcomes, as it will be shown in the following Sections. From an interpretative point of view, this means that the authority may act equivalently by increasing debt sensitivity $a$ beyond $a_{\text {min }}$, keeping $b$ fixed, or by adapting capital sensitivity $b$ into the interval $\left[b_{\min }, b_{\max }\right]$, keeping $a$ fixed, in order not to prevent convergent economic scenarios from occurring.

This situation is highlighted in figure 4, where the grey points represent possible combinations of parameters $a$ and $b$ at which the stationary states $E_{-}$ and $E_{+}$exist and are componentwise positive. Differently, the white points denote combinations of $a$ and $b$ at which no stationary state is present. We note that, with the considered parameter configuration, relations (11) and (13) boil down to the unique $b<b_{\max }$, being $b_{\min }<0$ for all considered values of $a$ in its range of variation. The figure underlines the trade-off between $a$ and $b$ to ensure the existence of $E_{+}$and $E_{-}$. Indeed, given a certain fixed level of capital responsiveness $b$, stationary states exist provided sufficiently high values of debt sensitivity $a$ are selected. Differently, given a certain fixed level of debt responsiveness $a$, stationary states exist provided sufficiently low values of capital sensitivity $b$ are selected. From an interpretative point of view, this means that the authority has the chance to manage with fiscal parameters by increasing $a$, by decreasing $b$, or by acting on both parameters, in order to allow the existence of equilibria. 


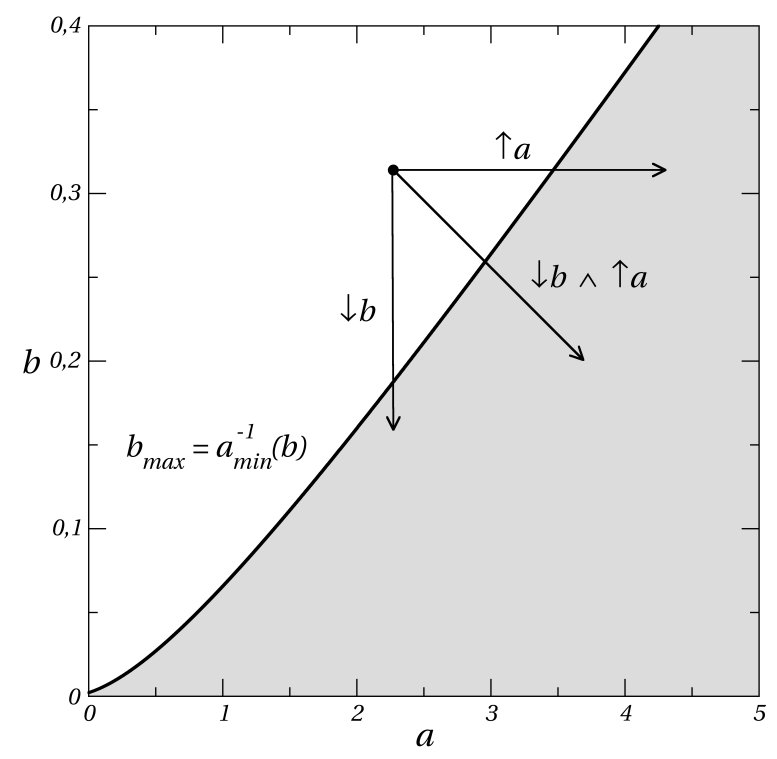

Figure 4. Parameter space where $b_{\max }$ (equivalently $a_{\min }^{-1}(b)$ ) is represented by the solid line. Parameters are $\gamma=0.15, \alpha=$ $0.25, \beta=0.99$ and $A=8$.

\section{LOCAL STABILITY}

In the present Section we provide local stability analysis of the stationary states $E_{-}$and $E_{+}$based on the study of eigenvalues of Jacobian matrix of the map (2) computed at $E_{-}$and $E_{+}$respectively. This methodological approach leads us to different conclusions with respect to the ones obtained in [7, where local stabilities are evaluated by means of the so called "arrows method" that, in our opinion, led the author to obtain misleading results. Indeed, as noted in [2], the "arrows method" gives only rough indications about dynamic behaviors in the neighborhood of a stationary state when applied to discrete time dynamical systems.

However, we avoid to derive analytical stability conditions for $E_{-}$and $E_{+}$, even if possible, due to burdensome computations (Jacobian matrices at $E_{ \pm}$, together with their traces and determinants, are provided in Appendix 7.3. We limit our analysis to exploit existence conditions provided in Proposition 3.1 as well as in Corollary 3.2 and to numerical computations in order to show the possibility for two bifurcation scenarios to occur and to highlight the role of policy parameters $a$ and $b$ in this context. 
Firstly, we mention that variations of $a$ and $b$ may cause saddle-node bifurcations, which is the basic mechanism that leads to the creation of the stationary states $E_{+}$and $E_{-}$. Occurrence of saddle-node is proved in the following Proposition exploiting, on the one hand, existence conditions from Corollary 3.2 . On the other hand, stability changes of stationary states are highlighted by means of numerical simulations at given parameter configurations. However, such stability patterns are found to be robust structures encountered at wide parameters configurations.

Proposition 4.1. Saddle-node bifurcation occurs as the parameter a (b) increases (decreases) crossing the threshold value $a_{\min }\left(b_{\max }\right)$, thus leading to the creation of the stationary states $E_{+}$and $E_{-}$.

Proof. By Corollary 3.2, at any parameters configuration for which $E_{+}$and $E_{-}$do not exist it results $a_{-}<a<a_{\min }$ or, equivalently, $b>b_{\max }$. Then, for example, the increase of $a$ up to $a_{\text {min }}$, or the decrease of $b$ down to $b_{\max }$, lead relations (10) and (11) to be satisfied with equal sign. This, in turn, implies that the condition (3) for existence of $E_{+}$and $E_{-}$is satisfied with equal sign as well, namely it holds $\sqrt{\delta_{2}}=\delta_{1}$. Hence, it results $r_{-}=r_{+}$, from which it follows that $E_{+}$and $E_{-}$coincides and merge at the tangent point of locus $\phi(k)$ and $\psi(k)$. This is shown in figure 5, where exemplary configurations of tangent locus $\phi$ and $\psi$ are provided. A further infinitesimal increase of $a$ beyond $a_{\mathrm{min}}$, or an infinitesimal decrease of $b$ below $b_{\max }$, implies that relation (8) is satisfied with strictly positive sign. Hence, stationary states $E_{+}$and $E_{-}$ exist and are distinct.

Moreover, from the numerical computations provided in figure 6 , it follows that the appearance of $E_{+}$and $E_{-}$is related to saddle-node bifurcation as $a$ varies, at the specific parameter configurations $b=0.013, \alpha=0.25, \gamma=0.15$, $\beta=0.99$ and $A=8$. In particular, left panels provide values of the characteristic polynomial $P_{+}(1)$ of matrix $J\left(E_{+}\right)$as a function of $a$. They show that, for a sufficiently small $\epsilon>0$, if $a=a_{\text {min }}+\epsilon, P_{+}(1)<0$ holds, thus implying that an eigenvalue of the Jacobian $J\left(E_{+}\right)$is real exits the stability interval $(-1,1)$ exceeding 1 . Similarly, right panels show values of the characteristic polynomial $P_{-}(1)$ of matrix $J\left(E_{-}\right)$as $a$ increases. They show that, for a sufficiently small $\epsilon>0$, if $a=a_{\min }+\epsilon, P_{-}(1)>0$ holds, thus implying that an eigenvalue of the Jacobian $J\left(E_{-}\right)$is real and enters the stability interval $(-1,1)$ on 1 side. 

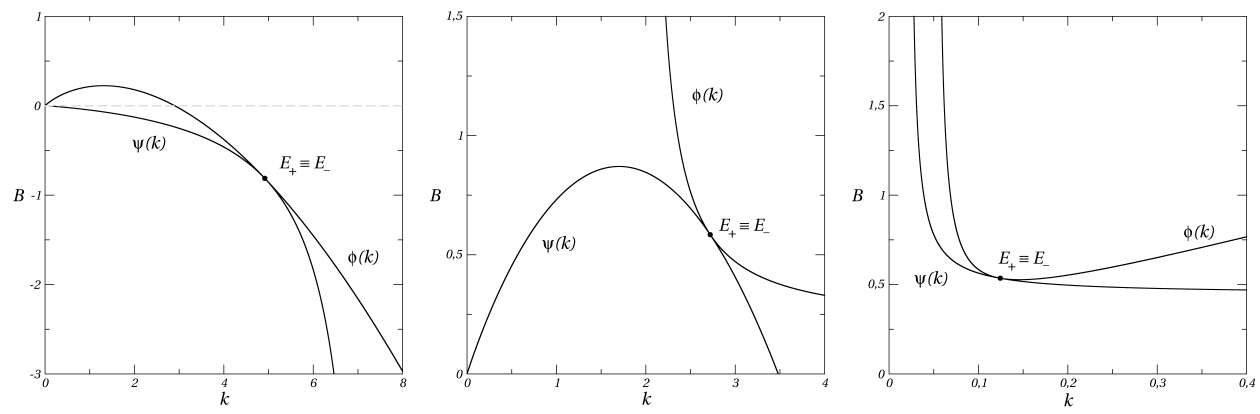

Figure 5 . Tangency points of locus where $E_{-} \equiv E_{+}$. Left. $a=a_{-}=0.259, b=b_{\max }=0.013, \gamma=0.15, \alpha=0.42$, $\beta=0.99, A=8$. Center. $a=a_{\min }=0.266, b=b_{\max }=0.013$, $\gamma=0.15, \alpha=0.25, \beta=0.99, A=8$. Right. $a=a_{\min }=0.853$, $b=b_{\max }=0.1, \gamma=0.15, \alpha=0.005, \beta=0.1, A=8$.

Analogous arguments can be discussed to prove the statement, formulated in terms of the parameter $b$, based on numerical computations performed as $b$ varies and at the specific parameter configuration $a=2, \alpha=0.25, \gamma=0.15$, $\beta=0.99$ to which figure (7) refers.
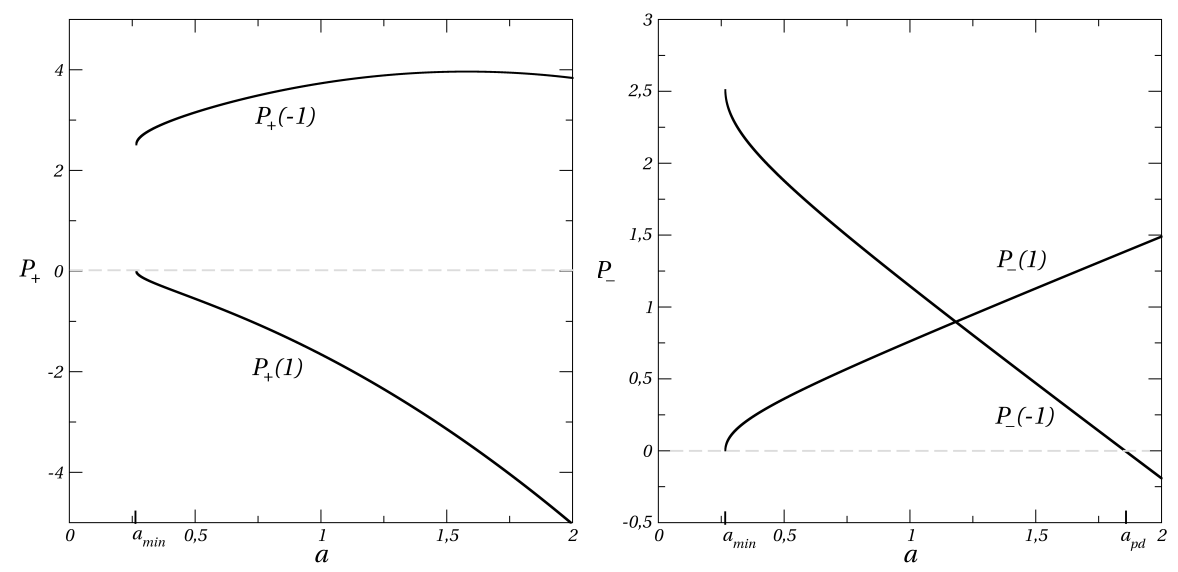

Figure 6. Characteristic polynomials at $E_{+}$(left) and at $E_{-}$ (right) as $a$ varies. Parameters are $b=0.013, \alpha=0.25, \gamma=$ $0.15, \beta=0.99$ and $A=8$. $a_{\min }$ and $a_{p d}$ correspond to saddlenode and period-doubling bifurcation thresholds respectively.

Remark 4.2. Proposition 4.1 claims the occurrence of dynamical scenarios that can be discussed as follows: at saddle-node bifurcation, an eigenvalue of 

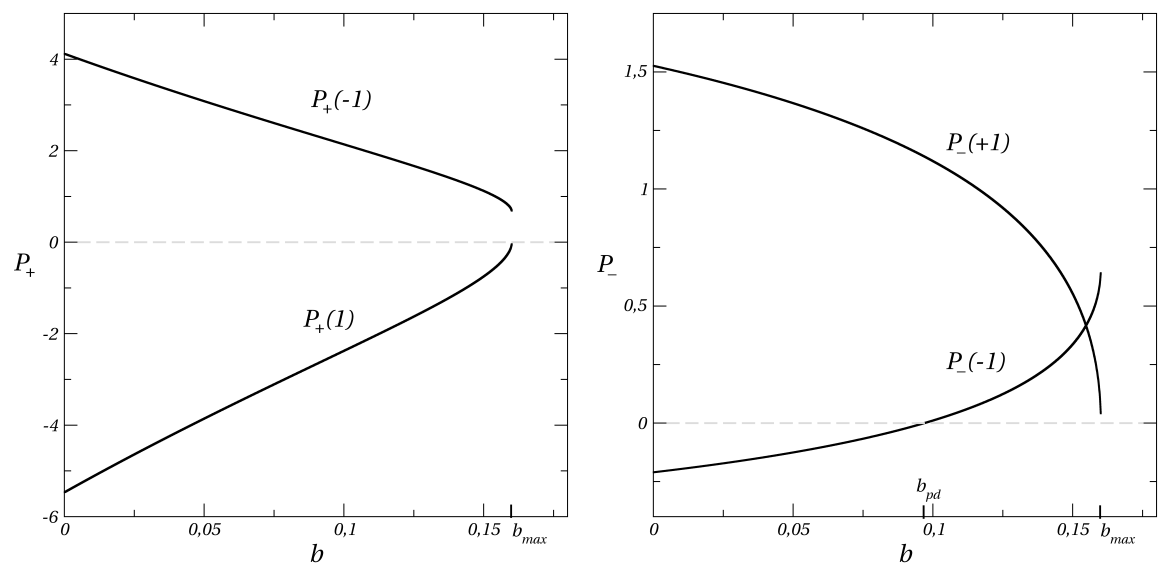

Figure 7 . Characteristic polynomials at $E_{+}$(left) and at $E_{-}$ (right) as $b$ varies. Parameters are $a=2, \alpha=0.25, \gamma=0.15$, $\beta=0.99$ and $A=8 . \quad b_{\max }$ and $b_{p d}$ correspond to saddle-node and period-doubling bifurcation thresholds respectively.

$J\left(E_{+}\right)$(let us say $\lambda_{+}^{(s n)}$ ) and an eigenvalue of $J\left(E_{-}\right)$(let us say $\lambda_{-}^{(s n)}$ ) both match value 1 . Then, a suitable and sufficiently small variation of a parameter (for example a small increase of a beyond $a_{\min }$, or a decrease of $b$ below $\left.b_{\max }\right)$, parts $E_{+}$and $E_{-}$one from the other. At the same time, this causes variations of eigenvalues $\lambda_{+}^{(s n)}$ and $\lambda_{-}^{(s n)}$ that, respectively, exit and enter the stability interval crossing 1. Moreover, just after their appearance, $E_{+}$and $E_{-}$are connected by a common invariant manifold. Along that manifold, $E_{+}$ is unstable, being the eigenvalue $\lambda_{+}^{(s n)}$ related with the eigenvector tangent to the manifold at $E_{+}$. At the same time, along the same manifold, $E_{-}$is stable, being the eigenvalue $\lambda_{-}^{(s n)}$ related to the eigenvector tangent to the manifold at $E_{-}$.

We remark also that saddle-node bifurcation can occur regardless of what happens to other eigenvalues of the Jacobian matrix computed at $E_{+}$and at $E_{-}$, the placement of which, with respect to the stability interval $(-1,1)$, follows from the sign of $P_{+}(-1)$ and $P_{-}(-1)$. In particular, it may occur that saddle-node bifurcation gives rise to $E_{+}$and $E_{-}$that are a saddle and a stable node respectively, with $P_{+}(-1)>0$ and $P_{-}(-1)>0$ (this is the case in figures 6 and 7). Differently, stationary states $E_{+}$and $E_{-}$can appear both unstable, more precisely an unstable node and a saddle respectively, with $P_{+}(-1)<0$ and $P_{-}(-1)<0$. 
Secondly, we found that stationary state $E_{-}$may undergo to period-doubling bifurcation as $a$ and $b$ vary. This is attested by the computations provided in figures 6 and 7 right panels, which show, respectively, that at an increase of $a$ or at a decrease of $b$ the characteristic polynomial $P_{-}(-1)$ passes from positive to negative numbers. We mention that this circumstance contradicts Proposition 2 in [7], where it is claimed that $E_{-}$is always stable. Again, due to burdensome computations, we prove the occurrence of period-doubling bifurcation limited to the exemplary case where the parameters set considered in [7] is selected. However, we remark that the occurrence of period-doubling bifurcation is a robust scenario encountered at a wide range of parameters sets.

Proposition 4.3. A threshold value $a_{p d}\left(b_{p d}\right)$ may exists such that, as the parameter a (b) varies crossing it, stationary state $E_{-}$undergoes to perioddoubling bifurcation.

Proof. Let us consider the parameter configuration $b=0.013, \alpha=0.25$, $\gamma=0.15$ and $A=8$ and let $a$ vary. As shown in figure 6, right panels, the threshold value $a_{p d} \approx 1.8548$ exists such that, if $a=a_{p d}$, the characteristic polynomial $P_{-}(-1)$ vanishes. Moreover, as $a$ increases crossing that threshold, the characteristic polynomial $P_{-}(-1)$ changes its value from positive to negative, implying that an eigenvalue of the Jacobian matrix of the map $T$ computed at $E_{-}$exits the stability region through the value -1, thus causing period-doubling bifurcation.

Analogous arguments can be discussed to prove the statement, formulated in terms of the parameter $b$, based on numerical computations performed at the parameters configuration $a=2, \alpha=0.25, \gamma=0.15$ and $A=8$ to which figure 7 refers. It follows that, as $b$ decreases crossing the threshold $b_{p d} \approx 0.0969$, an eigenvalue of the Jacobian $J\left(E_{-}\right)$exits the stability region through the value -1 , thus causing period-doubling bifurcation.

We summarize stability properties of $E_{-}$by means of the exemplary computations provided in figure 8, where regions in the $a-b$ parameter space, corresponding to different existence and stability regimes, are distinguished. In particular, the white points represent combinations of $a$ and $b$ such that no stationary states exist. Differently, the green and grey points correspond to values of $a$ and $b$ such that $E_{+}$and $E_{-}$exist and are componentwise positive. 


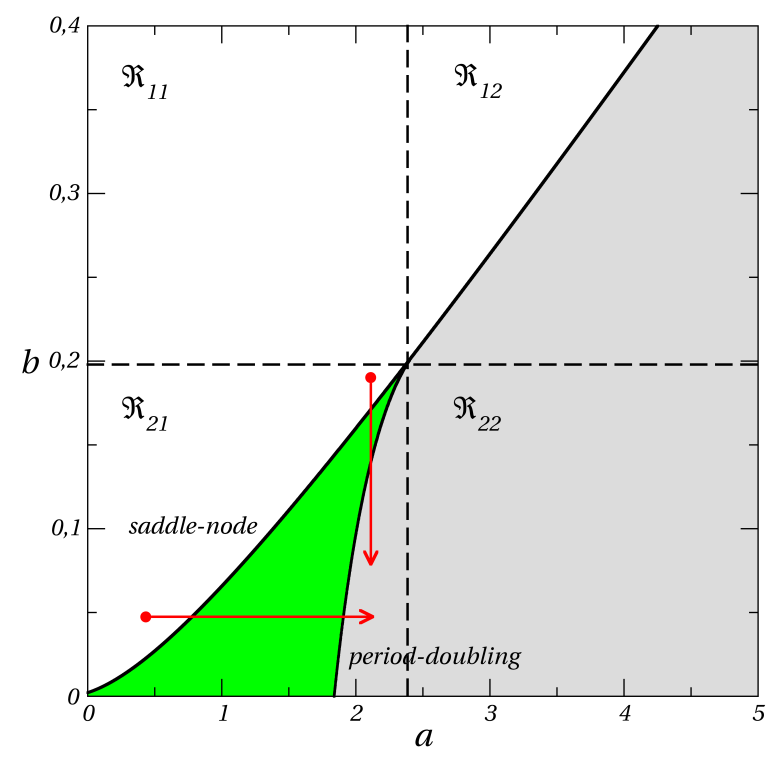

Figure 8. Parameter space where solid lines correspond to saddle-node and period-doubling thresholds. Parameters are $\alpha=0.25, \gamma=0.15, \beta=0.99$ and $A=8$.

Within the existence region, we further characterize those parameter sets for which $E_{-}$is locally asymptotically stable (green points) and those for which stationary states exist but are locally unstable (grey points).

From an interpretative point of view, the structure of existence and stability regions highlights that the fiscal policy authority has the chance to move sensitivity parameters $a$ and $b$ to allow the convergence of economy towards the stationary states $E_{-}$(stationary sustainability) given suitable initial conditions. Indeed, the parameter space can be parted in four regions, let us say $\Re_{i, j}$ with $i, j=1,2$, within each of which different actions are required in order to achieve control and predictability. In particular, from region $\Re_{11}$, a suitable decrease of $b$ within the interval $\left(b_{p d}, b_{\max }\right)$ as well as, from region $\Re_{22}$, a suitable decrease of $a$ within the interval $\left(a_{\min }, a_{p d}\right)$, led to the appearance of stationary states with stable $E_{-}$. Differently, from region $\Re_{21}$, the authority has the chance to achieve the goal equivalently by suitable adaptations of $a$ or of $b$, or of both. Finally, from region $\Re_{12}$, suitable adaptations of both $a$ and $b$ are needed in order to allow the presence of a stable stationary state, placing parameters configurations within the green region. Indeed, if only $a$ is 
sufficiently increased or $b$ is sufficiently decreased, the stationary states emerge but are an unstable node and a saddle.

We point out that figure 8 gives a first evidence of the ambiguous role of fiscal policies implemented by increasing debt responsiveness $a$ or by decreasing capital responsiveness $b$. Indeed, even if such policies determine the emergence of stationary states, they do not ensure the presence of a stable attractor. In particular, let us consider for instance the bifurcation path highlighted by the horizontal red arrow in the parameter space. Starting from the white part of region $\Re_{21}$, suitable increases of $a$ move parameters within the green region, thus leading to emergence of stationery states, one of which is a stable node. Hence, stationary sustainability can be achieved, given suitable initial conditions. However, whenever the increase is so important to bring $a$ beyond the period-doubling threshold $a_{p d}, E_{-}$is unstable and periodic as well as chaotic orbits may arise. Once again, dynamic sustainability, taking place along bounded trajectories, can still be achieved. In addition, as it will be shown in the following Section, if the increase of $a$ is extreme, the attractor could be unstable. In this case, divergence occurs anyway and non sustainability is the unique outcome of the model, regardless of initial conditions. Similar considerations can be discussed as $b$ is decreased following, for instance, the bifurcation path highlighted by the vertical red arrow in the parameter space. Indeed, starting from the white part of region $\Re_{21}$, the decrease of $b$ leads to the emergence of the stable stationary state $E_{-}$and then to its loss of stability. This may give rise to periodic and chaotic orbits. In both cases, stationary or dynamic sustainability can be achieved given suitable initial conditions. However, as it will be shown later, at further decreases of $b$, the attractor may lose its stability. In this case, again, divergence occurs anyway and non sustainability is the unique outcome of the model, regardless of initial conditions.

4.1. Periodic, complex dynamics and divergence. In the present Section we show that convergence and divergence are not unique outcomes of the model. Indeed, as already mentioned, stationary state $E_{-}$may undergo period-doubling bifurcation and, for a suitable variation of a parameter, that bifurcation may lead to the emergence of periodic orbits and, through the usual period doubling cascade, chaotic dynamics. Remarkably, we found that the route towards complex attractors can be followed varying fiscal parameters $a$ or $b$. Evidence of such an occurrence is provided in figure 9 that shows 
bifurcation diagrams of variables $k$ and $B$ (left and right panels respectively) as $a$ increases. Other parameters values correspond to those considered in [7]. The diagrams reveal that, increasing the value of $a$, the dynamic complexity of the attractor increases as well. In particular, a first increase of $a$ beyond the threshold $a_{\text {min }}$ determines the occurrence of saddle-node bifurcation and the emergence of stationary states. In this case, just after their appearance, $E_{+}$is a saddle and $E_{-}$is a stable node (both depicted by grey lines in the above mentioned figure). The topological equivalence of this scenario is maintained as $a$ increases until reaching the threshold $a_{p d}$, at which the stable node $E_{-}$loses its stability through period-doubling bifurcation, thus giving rise to a period 2 cycle. Moreover, as $a$ is further increased, the cycle enlarges its amplitude and, almost immediately after the period-doubling, it describes economic dynamics where positive and negative values of the debt $B$ alternate along the periodic trajectory. Then, as $a$ grows more and more, the loss of stability of the period 2 cycle and the rise of a stable period 4 cycle is caused. Finally, at a sufficiently high value of $a$, a global bifurcation occurs, thus leading to the stability loss of the attractor.

In figure 10 bifurcation diagrams of the variables $k$ and $B$ (left and right panels respectively) are provided as $b$ increases. This produces opposite behaviors of long run dynamics with respect to the case of increasing values of $a$, namely the dynamic complexity of the attractor is reduced. In particular, if $b$ is sufficiently low, no stable attractor is present and we remark that, in this case, stationary states $E_{+}$and $E_{-}$exist and are both unstable. In addition, at an increase of $b$, a period cycle acquires stability. When $b$ further raises, a sequence of period halving bifurcations takes place, thus determining the stability recovery of the stationary state $E_{-}$and, as soon as the threshold $b_{s n}$ is exceeded, the disappearance of $E_{+}$and $E_{-}$takes place through the saddle-node bifurcation.

Different bifurcation scenarios are provided in figures 11 and 12 , where long run behaviors of dynamic variables are shown as $a$ and $b$ increase respectively. We mention that these simulations were performed exploiting different fundamental parameters values with respect to that considered in [7]. The figures show the occurrence of period doubling cascade and the emergence of complex trajectories as $a$ is sufficiently increased and $b$ is sufficiently decreased. Also 

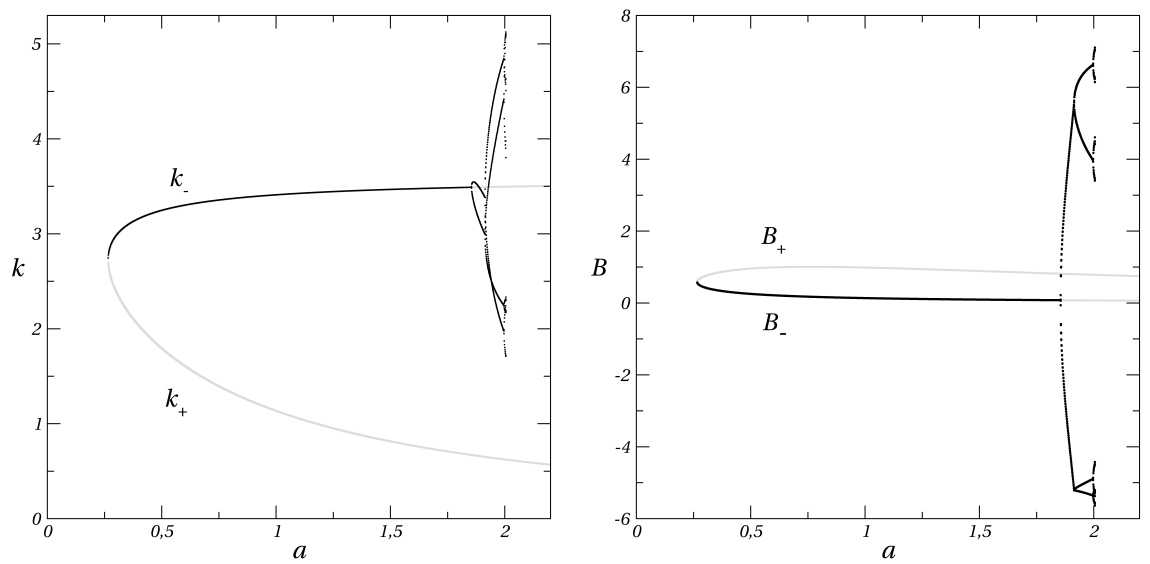

Figure 9. Bifurcation diagram of $k$ (left) and $B$ (right) as $a$ varies. Parameters are $b=0.013, \gamma=0.15, \alpha=0.25, \beta=0.99$, $A=8$.
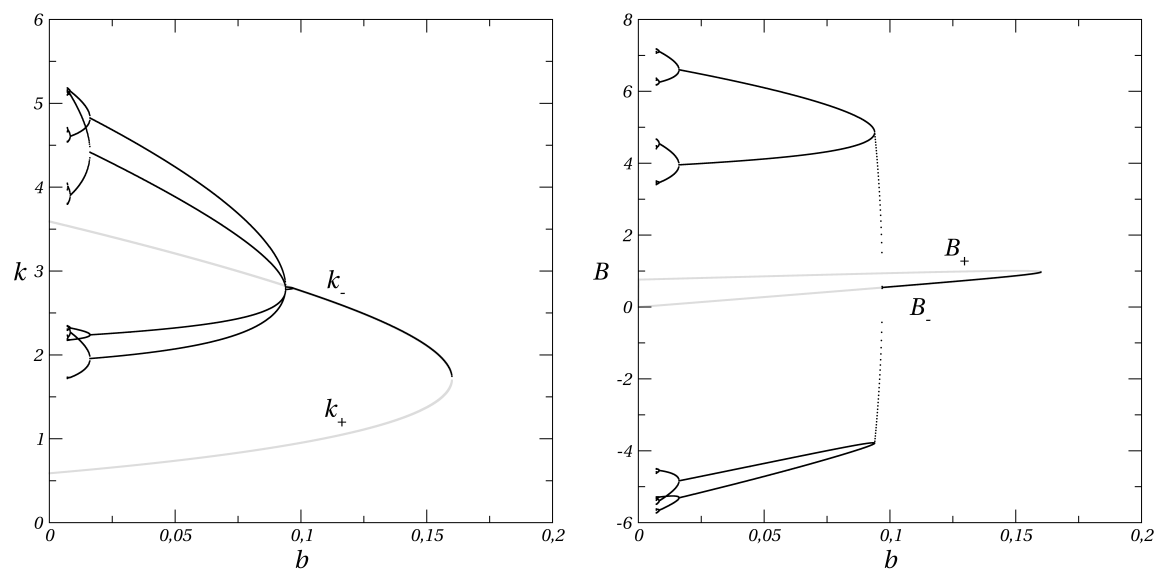

Figure 10. Bifurcation diagram of $k$ (left) and $B$ (right) as $b$ varies. Parameters are $a=2 ., \gamma=0.15, \alpha=0.25, \beta=0.99$, $A=8$.

in this case, the attractor may lose its stability at extremes values of $a$ and $b$ and only divergent dynamics are possible.

We end this Section with a comprehensive view on the role of policy parameters $a$ and $b$ in determining the presence of a stable attractor in the two exemplary simulations provided in figure 13 . Regions in the $a-b$ parameter space are distinguished in each panel depending on existence and dynamic properties of attractors. In detail, the white points represent $a$ and $b$ pairs for which no stationary state exist, while, the colored points, correspond to 

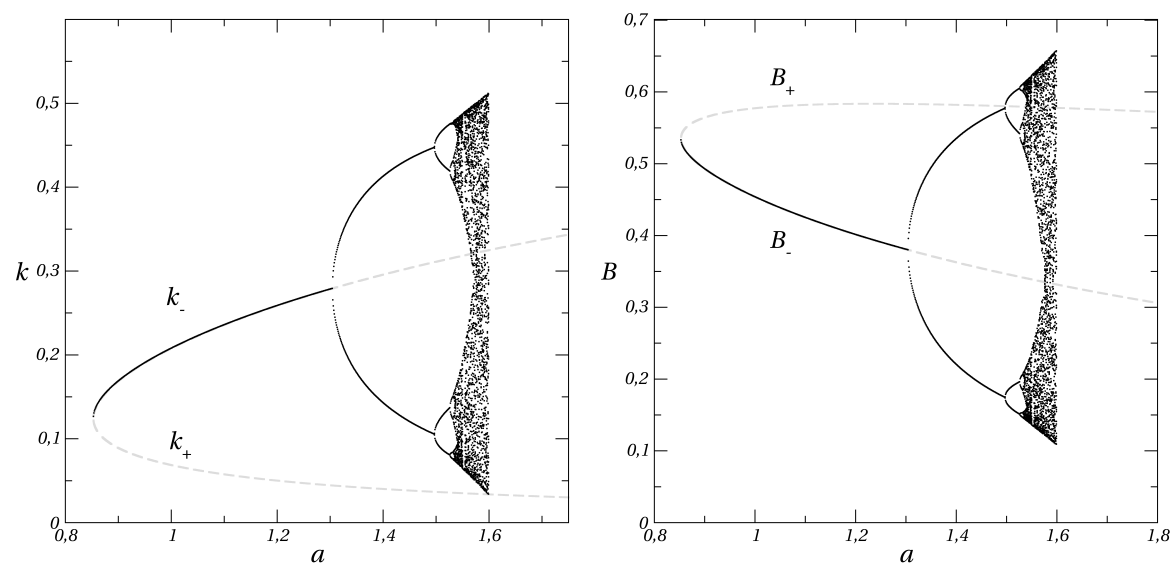

Figure 11. Bifurcation diagram of $k$ (left) and $B$ (right) as $a$ varies. Parameters are $b=0.1, \gamma=0.15, \alpha=0.005, \beta=0.1$, $A=8$.
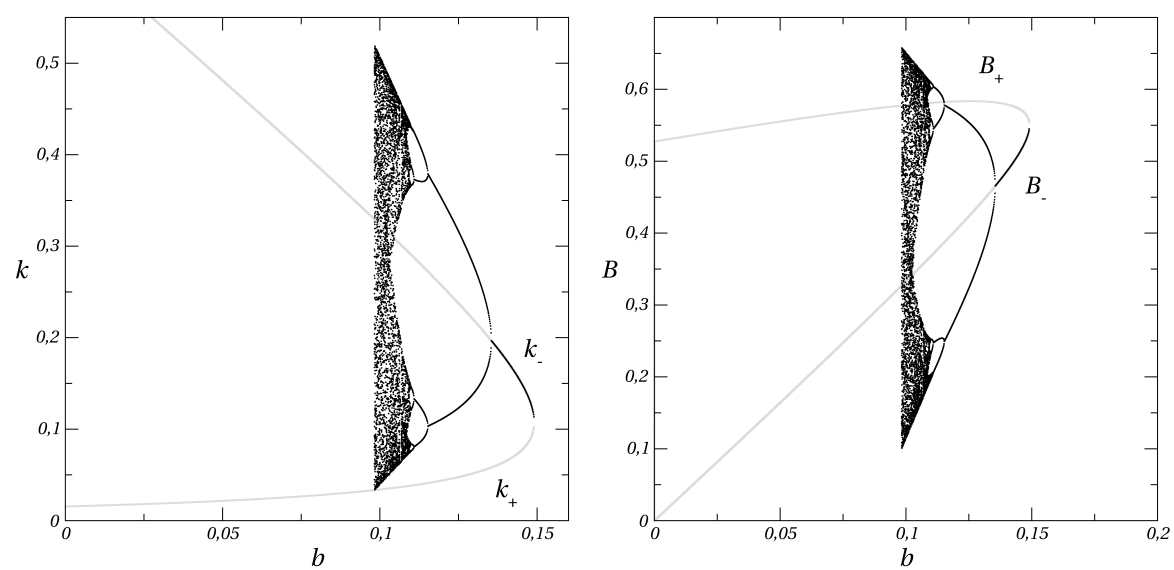

Figure 12. Bifurcation diagram of $k$ (left) and $B$ (right) as $b$ varies. Parameters are $a=1.59, \gamma=0.15, \alpha=0.005, \beta=0.1$, $A=8$.

existence of componentwise positive $E_{+}$and $E_{-}$. Within the existence regions, we further distinguish those $a$ and $b$ pairs, depicted in green, for which $E_{-}$is locally asymptotically stable and for which (static) sustainability can be achieved. Differently, blue points are pairs for which $E_{-}$is unstable and, around it, periodic of chaotic attractors are present. In this case dynamic sustainability can be achieved. Finally, the grey points denote those pairs for which divergence occurs anyway and non sustainability is the unique outcome. 

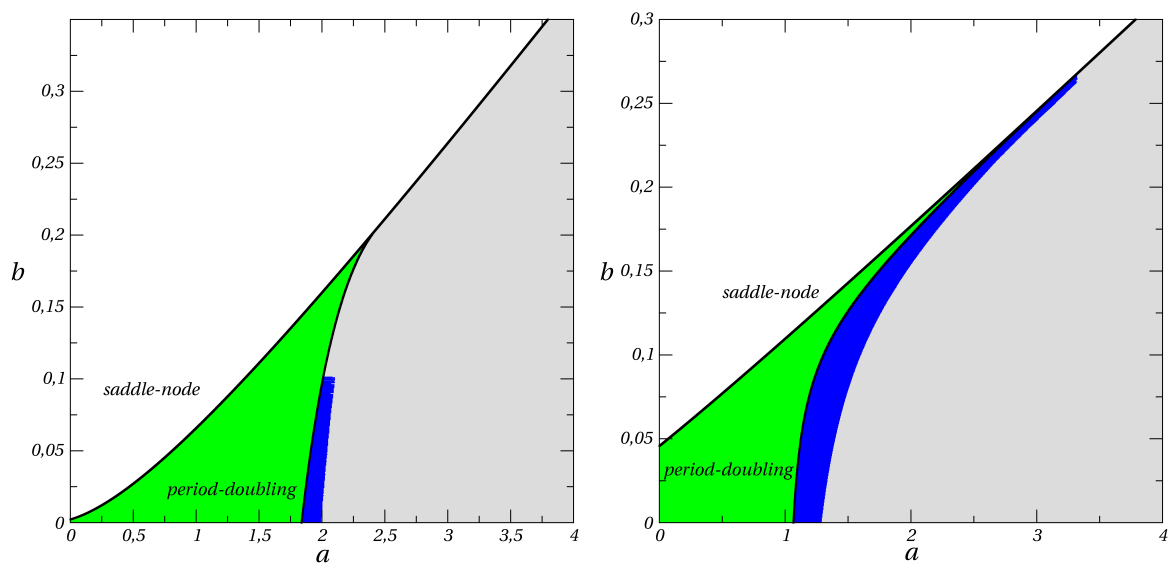

Figure 13. Parameter spaces. Left: $\alpha=0.25, \gamma=0.15$, $\beta=0.99$ and $A=8$. Right: $\alpha=0.005, \gamma=0.15, \beta=0.1$ and $A=8$.

\section{BASINS OF ATTRACTION}

In [7, the author argues that inherited debt and capital levels, representing the actual state of the economy and resulting from historical paths up to present time, contribute to determine incoming economic scenarios. Moreover, based on the idea that associates the occurrence of non divergent paths with the sustainability of fiscal policies, the author noted that "policymakers interested in the sustainability of various countries' debt need to look at not only its specific structural parameters but also their initial conditions $[, \ldots]$ that play a crucial role in determining the dynamics towards sustainability". In the present modeling framework, convergent dynamics can be achieved given the existence of a stable attracting set. Moreover, initial conditions must be included within the basin of the attractor, i.e. within the set of points of the phase space iterated in the long run towards the attractor under the action of the map $T$.

An example of the crucial role played by initial conditions is provided in figure 14, where the scenario obtained with the parameter configuration considered in [7] is represented through different scales of the phase space. In figure 14, white points denote the set of initial conditions from which diverging trajectories originate, while grey points represent the basin of attraction of the stable stationary state $E_{-}$. The left panel focuses on the shape of $E_{-}$'s basin in its neighborhood and shows that, given a certain level of initial debt, 
convergence towards the stationary state $E_{-}$is achieved at sufficiently high levels of initial capital stock, thus confirming findings by [7]. Nevertheless, we note that conclusions by [7] are limited to a local view. Indeed, a wider perspective on possible initial conditions shows that local behaviors of trajectories, varying initial conditions, is not maintained within the whole phase space. In particular, central and right panels of figure 14 provide enlarged views of the phase space and show that increasing values of initial capital levels have an ambiguous role. Indeed, if initial conditions are characterized by sufficiently high values of initial debt, initial capital levels for which convergence is achieved need to be sufficiently high without exceeding a certain upper threshold, beyond which initial conditions exit the basin of the attractor and divergence occurs again. Moreover, from the right panel, it follows that the increase of initial debt more and more reduces intervals of capital stock levels that place initial conditions within the basin of $E_{-}$, thus making convergence less likely to be achieved. The role of initial capital values is further highlighted in figure 15 that provides exemplary dynamic outcomes through time series obtained varying initial conditions. In the left panels, initial debt is fixed at a sufficiently low level $\left(B_{0}=1.5\right)$ and convergence is achieved as soon as initial capital $k_{0}$ is sufficiently high. Differently, the right panels, where initial debt is sufficiently high $\left(B_{0}=80\right)$, show that increasing values of $k_{0}$ lead at first to convergence and then to divergence.
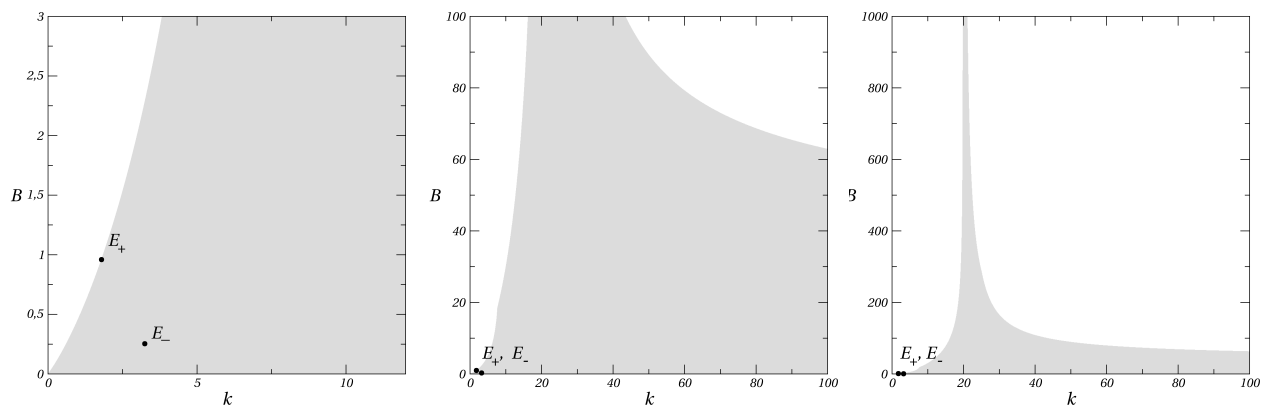

Figure 14. Phase space of the map $T$ in different scales. The gray points represent the basin of attraction of $E_{-}$while the white points represent the basin of divergence. Parameters are $a=0.5, b=0.013, \alpha=0.25, \gamma=0.15, \beta=0.99$ and $A=8$.

We remark that basins structures can be characterized by substantial differences with respect to the one observed above. One example can be given at 

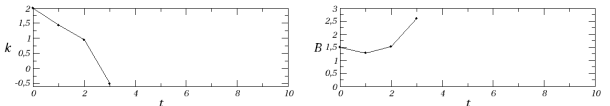

(a) $k_{0}=2, B_{0}=1.5$.
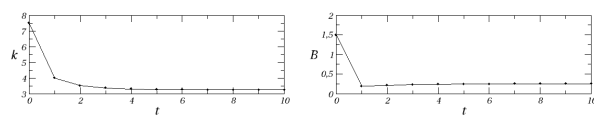

(b) $k_{0}=7.5, B_{0}=1.5$.
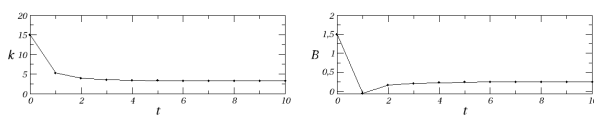

(c) $k_{0}=15, B_{0}=1.5$.

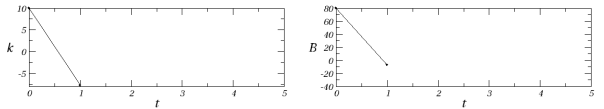

(d) $k_{0}=10, B_{0}=80$.
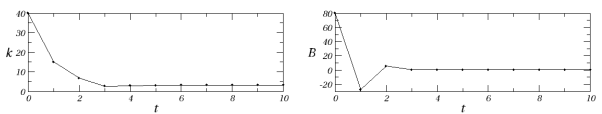

(e) $k_{0}=40, B_{0}=80$.

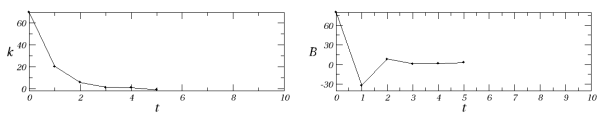

(f) $k_{0}=70, B_{0}=80$.

FiguRE 15. Trajectories $k_{t}$ and $B_{t}$ varying initial conditions: $k_{0}$ increases while $B_{0}$ is kept fixed at 1.5 (left) and 80 (right). Parameters are $a=0.5, b=0.013, \alpha=0.25, \gamma=0.15, \beta=0.99$ and $A=8$.

a slight variation of the parameter $a$, increased from the value 0.5 up to 1.8 . This generates a new scenario where differences from conclusions inferred by [7] are even more enhanced. Indeed, as shown in figure 16, the basin of stable $E_{-}$significantly shrinks in favor of the widening of the diverging trajectories basin. Approximately, the basin of stable $E_{-}$maintains a qualitatively similar structure with respect to the one observed in the previous scenario and similar remarks concerning initial conditions' variations apply. However, the same figure shows the presence of bubbles of the basin of divergent trajectories embedded within the basin of $E_{-}$. Hence, initial debt levels exist for which initial conditions are brought alternatively in and out the basin of $E_{-}$as initial capital values increase. This circumstance, highlighted in time series reported in figure 17. further emphasizes the ambiguous role of capital values.

By exploiting another configuration, characterized by different values of fundamental parameters, the basin of the stable $E_{-}$may reveal significant differences with respect to the ones observed in previous examples. This is the case of the dynamic scenario shown in figure 18, where increasing capital levels placing initial conditions in the basin of stable $E_{-}$with, alternatively, an ambiguous or an unambiguous role, are just those corresponding to a limited interval of intermediate initial debt levels. In addition, the same figure reveals that, at sufficiently low or sufficiently high debt levels, no initial capital exists 

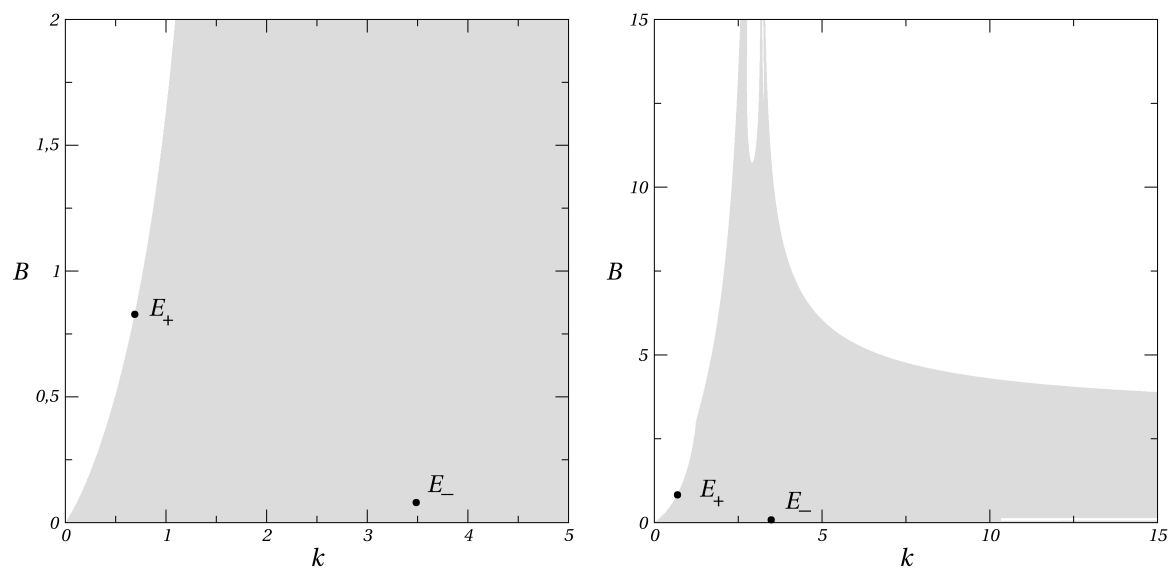

Figure 16. Phase space of the map $T$ in different scales. The gray points represent the basin of attraction of $E_{-}$while the white points represent the basin of divergence. Parameters are $a=1.8, b=0.013, \alpha=0.25, \gamma=0.15, \beta=0.99$ and $A=8$.

placing initial conditions within the basin of $E_{-}$, so that convergence may never occur.

\subsection{Influence of policy parameters on basins of attraction. Let us} focus on effects that fiscal policies have on basins of stable attractors. This is important in the present context since initial conditions, that result from historical outcomes to the present time, cannot be adjusted. Hence, the authority, with the aim to avoid divergence, should act on responsiveness parameters taking into account, at first, the effects of its policy in determining the presence of a stable attractors. The authority should also consider that its actions influence the extension and the shape of the basin of sustainable paths. This allows to address the problem of how to include initial conditions within the basin of sustainability by means of fiscal policy. Indeed, the variations of responsiveness $a$ and $b$ are capable of modifying outcomes of certain economic configurations, characterized by inherited debt and capital levels, that would have led to non sustainability if no action had been taken, even in the presence of a stable attractor. We remark that the possibility of including initial conditions within the basin of sustainability, after suitable variations of $a$ and $b$, have been noted in [7, which claims that convergence can be achieved if debt responsiveness $a$ is sufficiently increased or capital responsiveness $b$ is sufficiently decreased. On the contrary, we show that such policies may also 


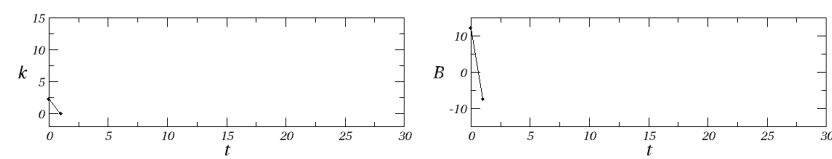

(a) $k_{0}=2.25, B_{0}=12$.
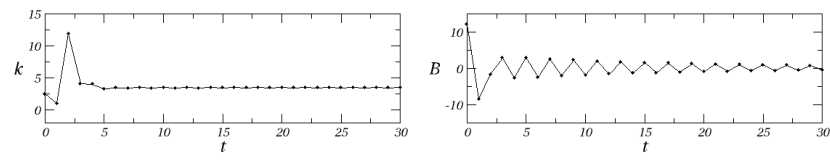

(b) $k_{0}=2.5, B_{0}=12$.
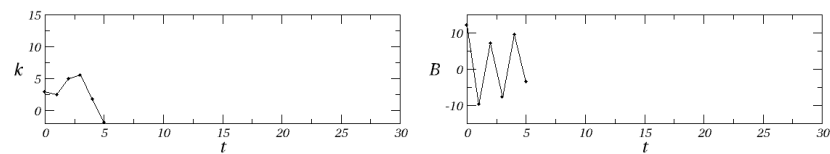

(c) $k_{0}=2.9, B_{0}=12$.
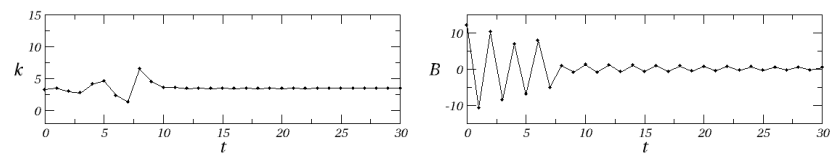

(d) $k_{0}=3.25, B_{0}=12$.
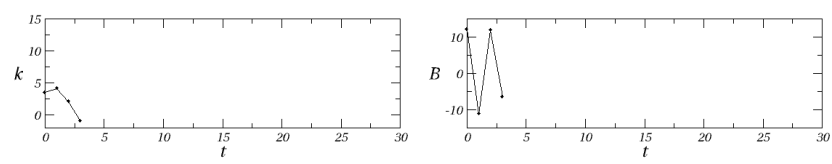

(e) $k_{0}=3.5, B_{0}=12$.

FIgURE 17. Trajectories $k_{t}$ and $B_{t}$ varying initial conditions: $k_{0}$ increases while $B_{0}$ is kept fixed at 12 . Parameters are $a=$ $1.8, b=0.013, \alpha=0.25, \gamma=0.15, \beta=0.99$ and $A=8$.

have opposite effects, and we identify two dynamic mechanisms, occurring as such policies are implemented, that contradict that statement.

The first mechanism consists in the fact that the basin of sustainability reduces, besides moving, as $a$ increases. This implies that the set of initial conditions, which bring dynamics to converge, does not widen. Hence, it may happen that one initial condition iterated towards non sustainable paths might not lead to convergence after high increases of $a$.

An example of this occurrence is highlighted in the numerical simulations provided in figure 19. Starting from the usual parameter configuration exploited in [7, the increase of $a$ from 0.5 up to 0.7 determines a new scenario 


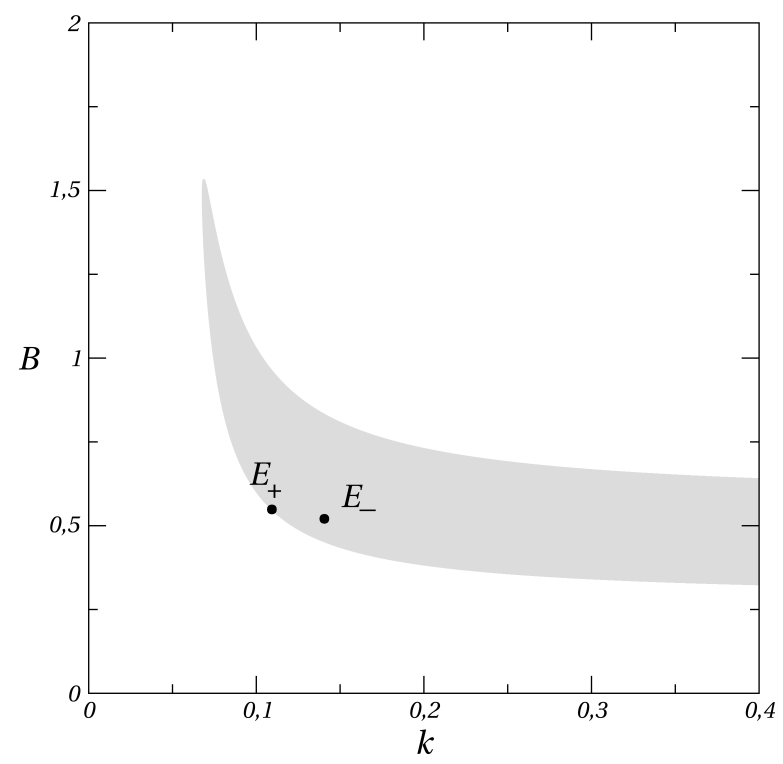

Figure 18. Phase space of the map $T$ where the gray points represent the basin of attraction of $E_{-}$while the white points represent the basin of divergence. Parameters are $a=0.86$, $b=0.1, \alpha=0.005, \gamma=0.15, \beta=0.1$ and $A=8$.

where the stationary state $E_{-}$is still stable and where a set of initial conditions, originally included within the basin of divergent trajectories, gets included within the basin of stable $E_{-}$after the variation. As an example, we show the different behaviors of a single initial condition $\left(k_{0}, B_{0}\right)=(5,7.5)$. In the first case, when $a=0.5$, the point $\left(k_{0}, B_{0}\right)$ gives rise to a divergent trajectory whereas, as $a$ increases at 0.7 , that point is included in the basin of $E_{-}$, thus leading to a convergent trajectory. However, if $a$ increases too much, at 1.8 in the right panel, it leaves the basin of $E_{-}$thus giving rise to divergence. Time series of trajectories starting from $\left(k_{0}, B_{0}\right)$ are provided at the bottom of the same figure 19 in the different cases considered in simulations.

The second mechanism that leads to contradict the above mentioned statement refers to the event in which, at sufficiently high values of $a$ or at sufficiently low values of $b$, no stable attractors might exist. This occurs, for example, in the last dynamic scenario considered when debt responsiveness $a$ is further increased. This is shown in figure (20), where changes of the basin of attractor, represented by the grey points of the phase space, are embedded with changes of the attractor itself, due to the influence of $a$ on its stability 

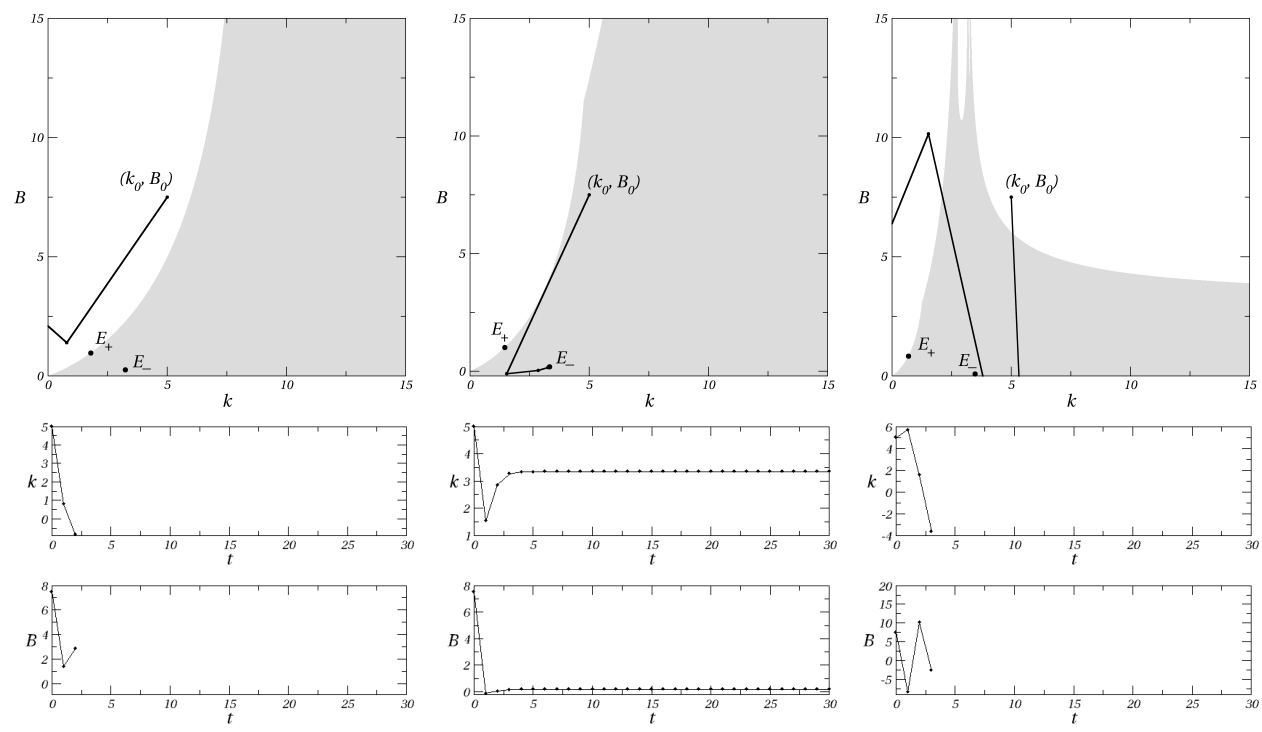

Figure 19. Phase space and basins of attractions at $a=0.5$ (left); $a=0.7$ (center); $a=1.8$ (right). Initial condition at $\left(k_{0}, B_{0}\right)=(5,7.5)$. Other parameters are $b=0.013, \gamma=0.15$, $\alpha=0.25, \beta=0.99, A=8$.

properties. The left panel (obtained at $a=1.87$ ) represents the case in which a stable period 2 cycle $C_{2}$, originated after the loss of stability of $E_{-}$through period-doubling bifurcation, is present and placed both at positive and negative levels of the debt. The basin of $C_{2}$ is disconnected and contains regions of initial conditions generating divergent trajectories. Such an occurrence is enhanced in the scenario showed in the central panel, obtained as $a$ has increased to the value 1.96 . Here, a period 4 cycle $C_{4}$, originated from $C_{2}$ after its loss of stability through a second period-doubling bifurcation, is present. The basin of $C_{4}$ is connected in its immediate neighborhood of and small variations of initial conditions give rise to trajectories converging towards it. However, the global shape of the basin of $C_{4}$ has an extremely complex and fractal structure that makes it intermingled with the basin of divergent trajectories. Because of this occurrence, infinitesimal variations of initial conditions lying sufficiently far from the cycle may generate, alternatively, convergent and divergent trajectories, thus bringing to the extreme the ambiguous role of varying initial capital levels. We also mention that the further increase of $a$ reduces even more the extension of the basin of the cycle $C_{4}$ in favor of the expansion of the basin of divergent trajectories, thus causing the loss of stability of the 
attractor due to a global bifurcation occurring as the attractor has a contact with the basin of divergent trajectories. This is the case reported in the right panel, where no stable attractor exists and divergence occurs regardless of initial conditions.
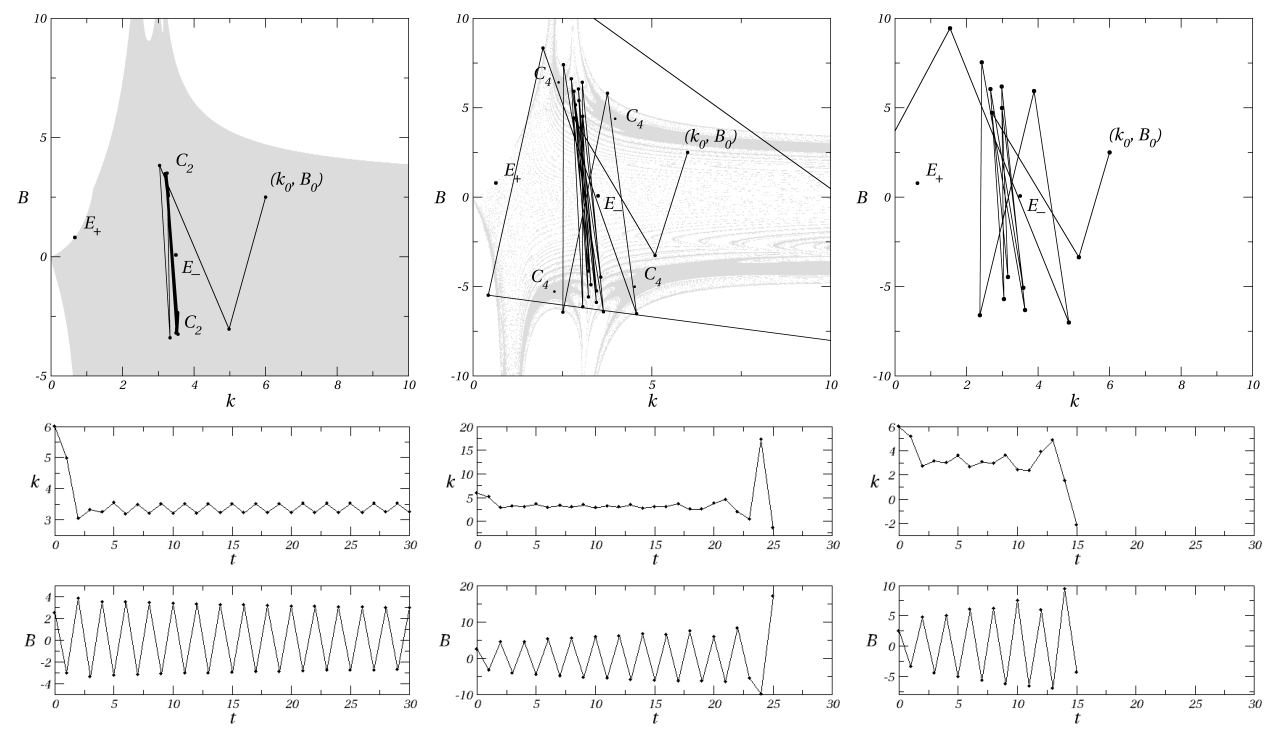

FiguRe 20. Phase space and basins of attractions at $a=1.87$ (left), $a=1.96$ (center) and $a=2$ (right). Initial condition at $\left(k_{0}, B_{0}\right)=(6,2.5)$. Other parameters are $b=0.013, \gamma=0.15$, $\alpha=0.25, \beta=0.99, A=8$.

A similar occurrence, where the stable attractors disappear as $a$ increases, is shown for a different parameters configuration in figure 21. As usual, the grey points of the phase space represent the basin of the stable attractor. In the left panel, where $a=0.9, E_{+}$is a saddle and $E_{-}$is a stable node. In the central panel, $a$ is increased up to the value 1.59 at which stationary states are both unstable and a chaotic attractor is present. Besides the structural changes of the attractors in the two mentioned configurations, the variation of $a$ determines the different shapes of their basins, thus varying the set of initial conditions leading to convergence. In the former case the trajectory diverges, whereas in the latter one it converges towards a chaotic attractor. Differently, in the right panel, a further rise of $a$ up to 1.65, caused a global bifurcation due to the contact of the attractor with its basin and hence to the disappearance of the stable attractor. Also in this scenario, divergence always happens regardless of initial conditions. As an example, we show the behavior 
of the trajectory starting from the initial condition $\left(k_{0}, B_{0}\right)=(0.1,0.25)$ in these cases, whose time series are provided at the bottom of the same figure 21 .
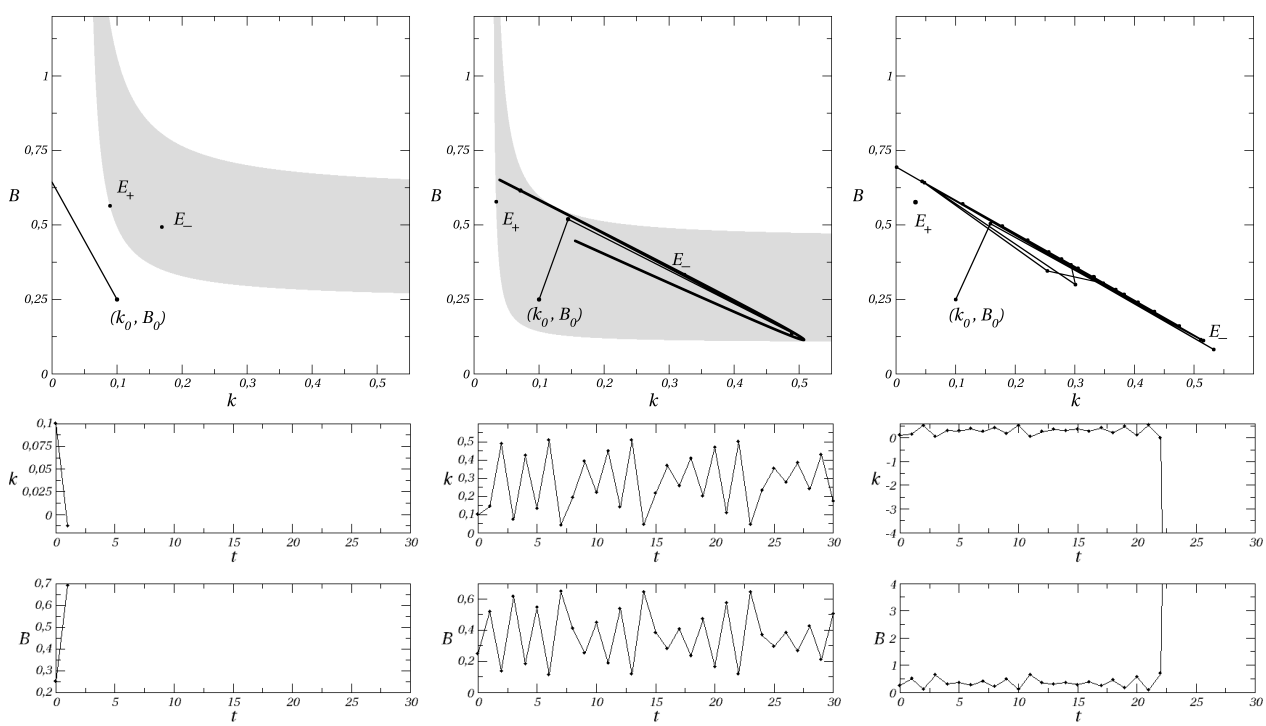

Figure 21. Phase space and basins of attractions at $a=0.9$ (left); $a=1.59$ (center); $a=1.65$ (right). Initial condition at $\left(k_{0}, B_{0}\right)=(0.1,0.25)$. Other parameters are $b=0.1, \gamma=0.15$, $\alpha=0.005, \beta=0.1, A=8$.

The structural variation of the basin of sustainability and the disappearance of the stable attractor may also be the consequence of decreasing capital responsiveness $b$. An example of this occurrence is provided in the sequence of dynamic scenarios presented in figure 22, showing numerical simulations performed at the fundamental parameters values exploited in [7]. The left panel represents a scenario where $b=0.0007$ at which the attractor is unstable and generic initial conditions generate divergent trajectories. The central panel is obtained at an increase of $b$ up to the value 0.008 , which is high enough to determine the presence of a stable period 8 cycle. Its basin is characterized by a fractal structure and it is extremely intermingled with the basin of divergent trajectories. As $b$ further increases at 0.09 , the basin of the attractor widens and, at the same time, the cycle $C_{8}$ has changed into the stable period 4 cycle $C_{4}$, as shown in the right panel. Finally, we mention that, as $b$ crosses the period-doubling threshold $b_{p d}$ and is maintained below the saddle-node threshold $b_{\max }$, the stationary state $E_{-}$retrieves its stability 

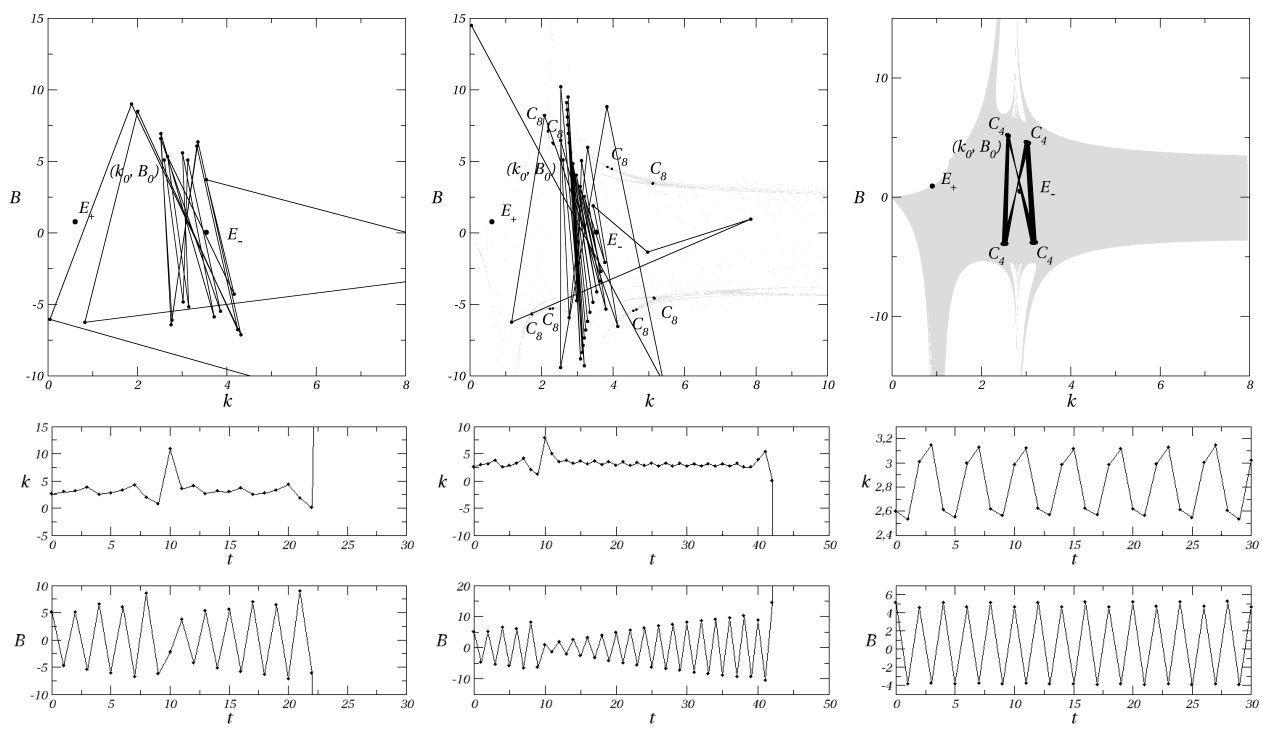

Figure 22. Phase space and basins of attractions at $b=$ 0.0007 (left), $b=0.008$ (center) and $b=0.09$ (right). Initial condition at $\left(k_{0}, B_{0}\right)=(2.6,5.1)$. Other parameters are $a=2, \alpha=0.25, \gamma=0.15, \beta=0.99$ and $A=8$.

\section{Conclusions}

We considered the overlapping generation model formulated in Dioikitopoulos (2018) placing within the theoretical framework that considers the problem of debt sustainability. The author, through hid analysis of the model, provided conditions for sustainability achievement in terms of debt and capital control channels, highlighting the role of initial conditions expressed in terms of inherited debt and capital levels. Our contribution has improved his study by means of rigorous and structured mathematical analysis performed through both analytical and numerical techniques. The analysis has revealed a wider spectrum of possible economic outcomes that might sometimes be opposed to the conclusions to which [7] came. In detail, we have derived analytical expressions for the stationary states of the model and we have provided necessary and sufficient conditions for their existence as well as for their componentwise positiveness, highlighting in this context the role of fiscal policy prescriptions implemented through debt and capital control channels. We have also deepened the influence of policy parameters on local stability properties of stationary states and we have revealed the possible occurrence of two bifurcation 
scenarios together with the consequent emergence of periodic and complex dynamics. Finally, we have reviewed the capability of fiscal policies in changing the fate of economic scenarios that would have led to non sustainable paths if no action had been taken by the authority.

Within the theoretical framework for debt sustainability here considered, further fiscal policy rules should be taken into account in future research.

Acknowledgement 6.1. The authors thank Professor Gian Italo Bischi for his helpful suggestions. The usual disclaimer applies.

\section{APPENDIX}

7.1. Proof of Proposition 3.1. Null variations of state variables occur at values of $k$ and $B$ that satisfy the following system of equations

$$
\left\{\begin{array}{l}
(s(1-\alpha)+s(b-\gamma)-b) y(k)-k+(a(1-s)-R(k)) B=0 \\
(R(k)-1-a) B+b y(k)=0
\end{array}\right.
$$

Part i).

Step 1. Stationary states.

Consider the cases $a(1-s)-R(k) \neq 0, R(k)-1-a \neq 0$ and $y(k) \neq 0$. Values of $B$ satisfying the first and the second equations in system (14) can be expressed in terms of $k$, respectively, as

$$
\phi(k)=\frac{(s(1-\alpha)+s(b-\gamma)-b) y(k)-k}{R(k)-a(1-s)}, \quad \psi(k)=\frac{b y(k)}{1+a-R(k)}
$$

For brevity's sake, let us introduce the shortcut $f:=s(1-\alpha)+s(b-\gamma)-b$. Observing that $y(k)=k R(k) / \alpha$, the condition $\phi(k)=\psi(k)$ leads to

$$
\frac{s(1-\alpha)+s(b-\gamma)-b}{b}=\frac{\alpha}{b R(k)}+\frac{R(k)-a(1-s)}{1+a-R(k)}
$$

This equation can be rewritten in terms of the second order polynomial $Z$ in the variable $r=R(k)$ as

$$
\begin{aligned}
& Z(r):=r^{2}(s(1-\alpha-\gamma)+b s)+ \\
& -r(\alpha+s(1-\alpha-\gamma)(1+a)-b(1-s))+\alpha(1+a)=0
\end{aligned}
$$

with discriminant

$$
\begin{aligned}
\Delta & =(\alpha+s(1-\alpha-\gamma)(1+a)-b(1-s))^{2}-4 \alpha(1+a)(s(1-\alpha-\gamma)+b s) \\
& =\delta_{1}^{2}-\delta_{2}
\end{aligned}
$$


and with roots

$$
\begin{aligned}
r_{ \pm}= & \frac{1}{2(s(1-\alpha-\gamma)+b s)}(\alpha+s(1-\alpha-\gamma)(1+a)-b(1-s) \pm \\
& \left. \pm \sqrt{(\alpha+s(1-\alpha-\gamma)(1+a)-b(1-s))^{2}-4 \alpha(1+a)(s(1-\alpha-\gamma)+b s)}\right) \\
= & \frac{1}{2(s(1-\alpha-\gamma)+b s)}\left(\delta_{1} \pm \sqrt{\delta_{1}^{2}-\delta_{2}}\right)
\end{aligned}
$$

Hence, provided that inverses $R^{-1}\left(r_{+}\right)$and $R^{-1}\left(r_{-}\right)$exist, equation (16) is satisfied both at $k_{+}:=R^{-1}\left(r_{+}\right)$and $k_{-}:=R^{-1}\left(r_{-}\right)$, where

$$
k_{ \pm}=\left(\frac{r_{ \pm}}{\alpha A(\gamma A)^{\frac{\gamma}{1-\gamma}}}\right)^{-\frac{1-\gamma}{1-\alpha-\gamma}}
$$

We then conclude that solutions of system (14), when exist, are the pairs $\left(k_{-}, B_{-}\right)$and $\left(k_{+}, B_{+}\right)$, with $B_{+}:=\psi\left(k_{+}\right)$and $B_{-}:=\psi\left(k_{-}\right)$, explicitly given by

$$
B_{ \pm}=\frac{b k_{ \pm} r_{ \pm}}{\alpha\left(1+a-r_{ \pm}\right)}
$$

Step 2. Existence and positiveness of $k_{+}$and $k_{-}$.

Values $k_{+}$and $k_{-}$exist and are positive if and only if $r_{-}$and $r_{+}$exist and are positive respectively. Both $r_{+}$and $r_{-}$exist provided that $\Delta=\delta_{1}^{2}-\delta_{2} \geq 0$ or, equivalently, if either $\delta_{1} \leq-\sqrt{\delta_{2}}$ or $\sqrt{\delta_{2}} \leq \delta_{1}$. Moreover, by noting that $0 \notin\left[r_{-}, r_{+}\right]$, being the value of the convex parabola $Z$ at $r=0$ positive (i.e. $Z(0)=\alpha(1+a)>0)$, either $r_{-}, r_{+}<0$ or $r_{-}, r_{+}>0$ hold. Hence, since $r_{-} \leq r_{+}$, the conditions $r_{-}>0$ and $r_{+}>0$ reduce to the unique $r_{-}>0$. Taking into account that $\delta_{2}>0$, it is easy to verify that $r_{-}>0$ together with $\Delta \geq 0$ are equivalent to $\sqrt{\delta_{2}} \leq \delta_{1}$, that proves condition (3).

Step 3. Positiveness of $B_{ \pm}$.

Provided that $k_{+}$and $k_{-}$exist and are positive, the values $B_{+}=\phi\left(k_{+}\right)$ and $B_{-}=\psi\left(k_{-}\right)$exist as well. Moreover, since under the same assumption $r_{+}, r_{-}>0$ also hold, from expressions (20) there follow that $B_{+}$and $B_{-}$are positive if and only if $r_{+}<1+a$ and $r_{-}<1+a$ respectively. By noting that $1+a \notin\left[r_{-}, r_{+}\right]$, being the value of the convex parabola $Z$ at $r=1+$ a positive (i.e. $Z(1+a)=b(1+a s)(1+a)>0)$, either $r_{-}, r_{+}<1+a$ or $r_{-}, r_{+}>1+a$. Hence, conditions $r_{-}, r_{+}<1+$ a reduce to the unique $r_{+}<1+a$. 
Let us show that $r_{+}<1+a$ if and only if $\delta_{1}<2(s(1-\alpha-\gamma)+b s)(1+a)$. Assume $r_{+}<1+a$. This relation can be rewritten, using (18), as

$$
\sqrt{\delta_{1}^{2}-\delta_{2}}<2(s(1-\alpha-\gamma)+b s)(1+a)-\delta_{1}
$$

which implies $0<2(s(1-\alpha-\gamma)+b s)(1+a)-\delta_{1}$. Conversely, assume $\delta_{1}<2(s(1-\alpha-\gamma)+b s)(1+a)$. This relation can be rewritten as

$$
\frac{\delta_{1}}{2(s(1-\alpha-\gamma)+b s)}<1+a
$$

since $0<s(1-\alpha-\gamma)+b s$. Hence, it holds

$$
\begin{aligned}
r_{-} & =\frac{\delta_{1}}{2(s(1-\alpha-\gamma)+b s)}-\frac{\sqrt{\delta_{1}^{2}-\delta_{2}}}{2(s(1-\alpha-\gamma)+b s)} \\
& <1+a-\frac{1}{2(b+f)} \sqrt{\delta_{1}^{2}-\delta_{2}} \\
& <1+a
\end{aligned}
$$

implying $r_{+}<1+a$, since $1+a \notin\left[r_{-}, r_{+}\right]$. This proves relation (7) and we conclude by noting that $2(s(1-\alpha-\gamma)+b s)(1+a)=\delta_{2} /(2 \alpha)$,

Part ii).

Consider the case $a(1-s)-R(k)=0$, which occurs at $k=k^{\prime}$ where

$$
k=\left(\frac{a(1-s)}{\alpha A(\gamma A)^{\frac{\gamma}{1-\gamma}}}\right)^{-\frac{1-\gamma}{1-\alpha-\gamma}}
$$

Note that $k^{\prime}>0 . k^{\prime}$ solves the first equation of the system (14) provided that

$$
a(1-s)(s(1-\alpha-\gamma)-b(1-s)) / \alpha=1
$$

whereas the second equation in (14) is satisfied at

$$
B^{\prime}=\frac{b y\left(k^{\prime}\right)}{1+a-R\left(k^{\prime}\right)}=\frac{b k^{\prime} a(1-s)}{\alpha(1+a s)}
$$

Note that $B^{\prime}>0$. Then, the pair $\left(k^{\prime}, B^{\prime}\right)$ is the unique componentwise positive solution of (14). We note that, under condition (22), it results $k^{\prime}=k_{-}$and $B^{\prime}=B_{-}$.

Finally, we mention that, in both cases $y(k)=0$ and $R(k)-1-a=0$, the system (14) has no solution.

\subsection{Proof of Corollary 3.2, Part i).}


Step 1. Values of a for which the stationary $E_{+}$and $E_{-}$exist. Values of a for which the condition $\sqrt{\delta_{2}} \leq \delta_{1}$ (that is (3)) is satisfied are those such that $\delta_{1}>0$ and $\delta_{2} \leq \delta_{1}^{2}$. Moreover, the equation $\delta_{2}=\delta_{1}^{2}$ can be rewritten as

$$
\begin{aligned}
& X(1+a)=s^{2}(1-\alpha-\gamma)^{2}(1+a)^{2}+ \\
& \quad-2 s(1+a)(\alpha(1+2 b-\alpha-\gamma)+b(1-s)(1-\alpha-\gamma))+(\alpha-b(1-s))^{2}=0
\end{aligned}
$$

where $X$ is a second order polynomial in the variable $1+a$. Polynomial $X$ has strictly positive discriminant

$$
\begin{aligned}
\Delta_{a}: & =4 s^{2}\left((\alpha(1+2 b-\alpha-\gamma)+b(1-s)(1-\alpha-\gamma))^{2}-(1-\alpha-\gamma)^{2}(\alpha-b(1-s))^{2}\right) \\
& =16 s^{2} \alpha b\left(\alpha b+(1-s)(1-\alpha-\gamma)^{2}+(\alpha+b(1-s))(1-\alpha-\gamma)\right)
\end{aligned}
$$

and roots given by $a_{+}+1$ and $a_{-}+1$, where $a_{+}$and $a_{-}$are reported in (9). Values $a_{+}$and $a_{-}$are both acceptable solutions of the equation $\sqrt{\delta_{2}}=\delta_{1}$ expressed in terms of $a$. Indeed, it can be easily verified that $\delta_{1}>0$ holds both at $a=a_{-}$and $a=a_{+}$. In addition, the function $\delta_{1}-\sqrt{\delta_{2}}$ is convex w.r.t. a, being

$$
\frac{d^{2}}{d a^{2}}\left(\delta_{1}-\sqrt{\delta_{2}}\right)=\frac{\sqrt{\alpha(s(1-\alpha-\gamma)+b s)}}{2(1+a)^{3 / 2}}>0
$$

Hence, relation $\delta_{1}-\sqrt{\delta_{2}} \geq 0$, which corresponds to (3) and ensures the existence of stationary states $E_{+}$and $E_{-}$, holds if and only if $a \notin\left(a_{-}, a_{+}\right)$and condition (8) is proved.

Step 2. Values of a for which the stationary states $E_{+}$and $E_{-}$exist and are componentwise positive.

In order to find values of a for which, relation $\delta_{1}<\delta_{2} /(2 \alpha)$ (that is (7)) hold, let us rewrite it in terms of the parameter a as

$$
a>\frac{\alpha-b(1-s)}{s(1+2 b-\alpha-\gamma)}-1:=a_{0}
$$

Through algebraic manipulations it can be verified that $a_{0}<a_{+}$. Indeed, by multiplying both terms $a_{0}$ and $a_{+}$by the positive term $1+2 b-\alpha-\gamma$, relation $a_{0}<a_{+}$is equivalent to

$\alpha-b(1-s)<\alpha\left(\frac{1+2 b-\alpha-\gamma}{1-\alpha-\gamma}\right)^{2}+b(1-s) \frac{1+2 b-\alpha-\gamma}{1-\alpha-\gamma}+\frac{1+2 b-\alpha-\gamma}{(1-\alpha-\gamma)^{2}} \sqrt{\Delta_{a}}$ 
A further step forward leads to

$\alpha\left(1-\left(\frac{1+2 b-\alpha-\gamma}{1-\alpha-\gamma}\right)^{2}\right)-b(1-s)\left(1+\frac{1+2 b-\alpha-\gamma}{1-\alpha-\gamma}\right)<\frac{1+2 b-\alpha-\gamma}{(1-\alpha-\gamma)^{2}} \sqrt{\Delta_{a}}$

which is always satisfied since $1<\frac{1+2 b-\alpha-\gamma}{1-\alpha-\gamma}$ and $\Delta_{a} \geq 0$.

To conclude, we proved that $\sqrt{\delta_{2}} \leq \delta_{1}$ is equivalent to $a \notin\left(a_{-}, a_{+}\right)$and $\delta_{1}<\delta_{2} /(2 \alpha)$ is equivalent to $a_{0}<a$. Hence, considering that $a_{0}<a_{+}$, the two relations hold together if and only if $a_{+} \leq a$, and condition 10) follows. Part ii).

Step 1. Values of $b$ for which the stationary $E_{+}$and $E_{-}$exist. Taking into account that $\delta_{2}>0$, values of $b$ for which the condition $\sqrt{\delta_{2}} \leq \delta_{1}$ (that is (3)) is satisfied are those such that $0<\delta_{1}$ and $\delta_{2} \leq \delta_{1}^{2}$ hold. In particular, the relation $0<\delta_{1}$, expressed in terms of parameter $b$, can be rewritten as

$$
b<b_{0}:=\frac{\alpha+s(1-\alpha-\gamma)(1+a)}{1-s}
$$

while relation $\delta_{2} \leq \delta_{1}^{2}$ can be rearranged in the following equivalent form

$$
\begin{array}{r}
Z(b)=(1-s)^{2} b^{2}-(2 \alpha(1-s)+2 s(1-\alpha-\gamma)(1-s)(1+a)+4 \alpha s(1+a))+ \\
+(\alpha-s(1-\alpha-\gamma)(1+a))^{2} \geq 0
\end{array}
$$

where $Z$ is a second order polynomial in $b$ with strictly positive discriminant and with roots

$$
\begin{aligned}
b_{ \pm}=\frac{\alpha}{1-s} & +\frac{s(1-\alpha-\gamma)(1+a)}{1-s}+\frac{2 \alpha s(1+a)}{(1-s)^{2}} \pm \\
& \pm \frac{2}{(1-s)^{2}} \sqrt{\alpha s(1+a)(1+a s)(\alpha+(1-s)(1-\alpha-\gamma))}
\end{aligned}
$$

Hence, $Z(b) \geq 0$ holds whenever $b \leq b_{-}$or $b_{+} \leq b$. However, only the values of $b$ such that $b \leq b_{-}$are acceptable for the relation $\sqrt{\delta_{2}} \leq \delta_{1}$ to be satisfied. Indeed, let us show that the occurrence of $b_{+} \leq b$ implies $\delta_{1}<0$ :

$$
\begin{aligned}
\delta_{1} & =\alpha+s(1-\alpha-\gamma)(1+a)-b(1-s) \\
& \leq \alpha+s(1-\alpha-\gamma)(1+a)-b_{+}(1-s) \\
& =-\frac{2 \alpha s(1+a)}{1-s}-\frac{2}{(1-s)} \sqrt{\cdots}<0
\end{aligned}
$$


Differently, relation $b_{-}<b_{0}$ always holds. To show this let us rearrange that relation as

$$
\frac{2 \alpha s(1+a)}{(1-s)^{2}}-\frac{2}{(1-s)^{2}} \sqrt{\alpha s(1+a)(1+a s)(\alpha+(1-s)(1-\alpha-\gamma))}<0
$$

or, equivalently, as

$$
\alpha s(1+a)<\sqrt{\alpha s(1+a)(1+a s)(\alpha+(1-s)(1-\alpha-\gamma))}
$$

Since both terms in this last relations are positive, we obtain equivalent relation by rising both terms to square. Then we get

$$
\alpha^{2} s^{2}(1+a)^{2}<\alpha s(1+a)(1+a s)(\alpha+(1-s)(1-\alpha-\gamma))
$$

that leads to

$$
-\alpha<(1+a s)(1-\alpha-\gamma)
$$

which is always satisfied. To conclude, we proved that relation $b<b_{-}$is necessary and sufficient condition for the relation (3) to hold, that is for stationary states $E_{-}$and $E_{+}$to exist.

Step 2. Values of $b$ for which the stationary $E_{+}$and $E_{-}$exist and are componentwise positive.

In order to find values of $b$ for which the condition $\delta_{1}<\delta_{2} /(2 \alpha)$ (that is (7)) holds, let us rewrite it in terms of the parameter $b$ as

$$
b>b_{\min }:=\frac{\alpha-s(1-\alpha-\gamma)(1+a)}{1-s+2 s(1+a)}
$$

To conclude, we proved that $\sqrt{\delta_{2}} \leq \delta_{1}$ is equivalent to $b \leq b_{-}$and $\delta_{1}<\delta_{2} /(2 \alpha)$ is equivalent to $b>b_{\min }$. Hence, the two relations hold together if and only if $b_{\min }<b \leq b_{-}$, and condition (13) follows.

7.3. Jacobian matrices at $E_{-}$and $E_{+}$and stability conditions. It results that

$$
J\left(E_{ \pm}\right)=\left(\begin{array}{cc}
f \frac{r_{ \pm}}{1-\gamma}+\frac{1-\alpha-\gamma}{1-\gamma} \frac{b r_{ \pm}^{2}}{\alpha\left(1+a-r_{ \pm}\right)} & a(1-s)-r_{ \pm} \\
\frac{b r_{ \pm}}{1-\gamma}-\frac{1-\alpha-\gamma}{1-\gamma} \frac{b r_{ \pm}^{2}}{\alpha\left(1+a-r_{ \pm}\right)} & r_{ \pm}-a
\end{array}\right)
$$


with traces and determinants given by

$$
\begin{aligned}
\operatorname{tr} J\left(E_{ \pm}\right) & =\frac{r_{ \pm}}{1-\gamma}\left(s(1-\alpha-\gamma)-b(1-s)+\frac{1-\alpha-\gamma}{\alpha\left(1+a-r_{ \pm}\right)} b r_{ \pm}\right) \\
\operatorname{det} J\left(E_{ \pm}\right) & =\frac{r_{ \pm}}{1-\gamma}\left(r_{ \pm}-a\right)(s(1-\alpha-\gamma)+b s)+a s \frac{r_{ \pm}}{1-\gamma}\left(b-\frac{1-\alpha-\gamma}{1+a-r_{ \pm}} r_{ \pm}\right)
\end{aligned}
$$

where $r_{ \pm}$are given in (18).

We recall that a stationary state, let us consider $E_{+}$for instance, is locally asymptotically stable if and only if both eigenvalues of the Jacobian computed at $E_{+}$are included in the unitary circle in the complex plane. The same stability conditions can be equivalently reformulated in terms of the characteristic polynomial of $J\left(E_{+}\right)$defined by

$$
P_{+}(\lambda)=\lambda^{2}-\operatorname{tr} J\left(E_{+}\right)+\operatorname{det} J\left(E_{+}\right)
$$

as $P_{+}(1)>0, P_{+}(-1)>0$ and $\operatorname{det} J\left(E_{+}\right)<1$.

Analogous stability conditions hold for stationary state $E_{-}$, expressed in terms of the characteristic polynomial $P_{-}(\lambda)$ of $J\left(E_{-}\right)$.

\section{REFERENCES}

[1] David A. Aschauer. Is public expenditure productive? Journal of monetary economics, 23(2):177-200, 1989.

[2] Costas Azariadis. Intertemporal macroeconomics. 1993.

[3] Henning Bohn. The behavior of us public debt and deficits. the Quarterly Journal of economics, 113(3):949-963, 1998.

[4] Hafedh Bouakez, Michel Guillard, and Jordan Roulleau-Pasdeloup. Public investment, time to build, and the zero lower bound. Review of Economic Dynamics, 23:60-79, 2017.

[5] Charles Brendon and Giancarlo Corsetti. Coeure survey: Fiscal and monetary policies after the crises. JCEPR Discussion Paper No. DP11088. Available at SSRN: https://ssrn.com/abstract=2726568, 2016.

[6] Peter A. Diamond. National debt in a neoclassical growth model. The American Economic Review, 55(5):1126-1150, 1965.

[7] Evangelos V. Dioikitopoulos. Dynamic adjustment of fiscal policy under a debt crisis. Journal of Economic Dynamics and Control (to appear), 2018.

[8] M. Ishaq Nadiri and Theofanis P. Mamuneas. The effects of public infrastructure and r\&d capital on the cost structure and performance of u.s. manufacturing industries. The Review of Economics and Statistics, 76(1):22-37, 1994.

[9] Pierre M. Pestieau. Optimal taxation and discount rate for public investment in a growth setting. Journal of Public Economics, 3(3):217-235, 1974.

[10] Paul A. Samuelson. The pure theory of public expenditure. The review of economics and statistics, pages 387-389, 1954. 
[11] Jean Tirole. Asset bubbles and overlapping generations. Econometrica: Journal of the Econometric Society, pages 1499-1528, 1985.

[12] Stephen J. Turnovsky and Walter H. Fisher. The composition of government expenditure and its consequences for macroeconomic performance. Journal of Economic Dynamics and Control, 19(4):747-786, 1995. 